\title{
Search for $Z^{\prime}$ resonances decaying to $t \bar{t}$ in dilepton + jets final states in $p p$ collisions at $\sqrt{s}=7 \mathrm{TeV}$
}

\author{
S. Chatrchyan et al.* \\ (CMS Collaboration) \\ (Received 14 November 2012; published 3 April 2013)
}

\begin{abstract}
A search for resonances decaying to top quark-antiquark pairs is performed using a dilepton + jets data sample recorded by the CMS experiment at the LHC in $p p$ collisions at $\sqrt{s}=7 \mathrm{TeV}$ corresponding to an integrated luminosity of $5.0 \mathrm{fb}^{-1}$. No significant deviations from the standard model background are observed. Upper limits are presented for the production cross section times branching fraction of top quark-antiquark resonances for masses from 750 to $3000 \mathrm{GeV}$. In particular, the existence of a leptophobic topcolor particle $Z^{\prime}$ is excluded at the $95 \%$ confidence level for resonance masses $M_{Z^{\prime}}<1.3 \mathrm{TeV}$ for $\Gamma_{Z^{\prime}}=0.012 M_{Z^{\prime}}$, and $M<1.9 \mathrm{TeV}$ for $\Gamma_{Z^{\prime}}=0.10 M_{Z^{\prime}}$.
\end{abstract}

DOI: 10.1103/PhysRevD.87.072002

PACS numbers: 13.85.Rm, 12.60.Cn, 14.65.Ha

\section{INTRODUCTION}

Electroweak symmetry breaking is a cornerstone for the understanding of particle physics. However, despite the spectacular phenomenological success of the standard model (SM), and the recent observation of a new boson at the Large Hadron Collider (LHC) [1,2], the precise mechanism of electroweak symmetry breaking remains unknown. Various new models have been proposed to explain this mechanism. One such class of models, topcolorassisted technicolor (TC2) [3-5], provides a dynamical explanation for electroweak symmetry breaking and flavor symmetry breaking, giving masses to the weak gauge bosons and fermions. Under one of the scenarios of TC2, a heavy boson $Z^{\prime}$ is predicted with preferential couplings to the third quark generation and with no significant couplings to the leptons ("leptophobic").

Direct searches for massive resonances that decay preferentially to top quark-antiquark $(t \bar{t})$ pairs are currently feasible only at hadron colliders. Experiments seek to observe an excess beyond that predicted by the SM, typically in the distribution of the invariant mass of the $t \bar{t}$ decay products. Searches in $p \bar{p}$ collisions at the Tevatron and the early searches in $p p$ collisions at the LHC by the ATLAS experiment have excluded a narrow-width, leptophobic $Z^{\prime}$ with a mass lower than $900 \mathrm{GeV} \mathrm{[6-8].} \mathrm{The} \mathrm{searches} \mathrm{by} \mathrm{the}$ Compact Muon Solenoid (CMS) experiment at the LHC have excluded a narrow-width, leptophobic $Z^{\prime}$ in the mass range $1.3-1.5 \mathrm{TeV}$ and in a narrow window around $1 \mathrm{TeV}$ $[9,10]$. This paper describes a search for a $Z^{\prime} \rightarrow t \bar{t}$ resonance in $p p$ collisions in the $2 \ell+2 \nu+$ jets final state, where $\ell$ is an electron $(e)$ or a muon $(\mu)$. This is the first

*Full author list given at the end of the article.

Published by the American Physical Society under the terms of the Creative Commons Attribution 3.0 License. Further distribution of this work must maintain attribution to the author(s) and the published article's title, journal citation, and DOI. search for topcolor leptophobic $Z^{\prime}$ in final states involving two leptons. The data sample corresponds to a total integrated luminosity of $5.0 \mathrm{fb}^{-1}$ [11] at $\sqrt{s}=7 \mathrm{TeV}$ collected by the CMS detector in 2011 .

\section{THE CMS DETECTOR}

The central feature of the CMS apparatus is a superconducting solenoid, $13 \mathrm{~m}$ in length and $6 \mathrm{~m}$ in diameter, which provides an axial magnetic field of $3.8 \mathrm{~T}$. The bore of the solenoid is surrounded by various particle detection systems. Charged particle trajectories are measured by a silicon pixel and strip tracker, covering $0<\phi \leq 2 \pi$ in azimuth and $|\eta|<2.5$, where the pseudorapidity $\eta$ is defined as $\eta=-\ln [\tan (\theta / 2)]$, and $\theta$ is the polar angle of the trajectory of the particle with respect to the counterclockwise-beam direction. A crystal electromagnetic calorimeter and a brass/scintillator hadronic calorimeter surround the tracking volume. The calorimeter provides high-resolution energy measurement of electrons. Muons are measured in gas-ionization detectors embedded in the steel flux return yoke outside the solenoid. The detector is nearly hermetic, which facilitates the measurement of energy balance in the plane transverse to the beam direction. A two-tier trigger system selects the most interesting $p p$ collision events for use in physics analysis. A more detailed description of the CMS detector can be found in Ref. [12].

\section{EVENT RECONSTRUCTION}

In the $Z^{\prime} \rightarrow t \bar{t}$ search, a $t \bar{t}$ decay topology is used where each top quark decays to a $W$ boson and a $b$ quark, and subsequently each $W$ boson decays into a lepton and a neutrino. The signature for such an event is two oppositely charged, isolated leptons with high transverse momenta $\left(p_{\mathrm{T}}\right)$, large momentum imbalance due to two undetected neutrinos, and at least two jets. Events are required to pass a trigger requiring at least two high- $p_{\mathrm{T}}$ isolated leptons and 
are separated into three channels based on lepton flavor: $e e, \mu \mu$, and $e \mu$. The principal sources of background are $\mathrm{SM} t \bar{t}, Z / \gamma^{*} \rightarrow \ell \bar{\ell}$ [Drell-Yan (DY)], single-top quark, and diboson $(W W, W Z$, and $Z Z)$ production. Other minor contributions are from $W \rightarrow \ell \nu$ and multijet production. Electrons, muons, jets, and the momentum imbalance are reconstructed using a particle-flow algorithm [13]. The negative of the vector sum of the momenta of all reconstructed particles in the plane transverse to the beams is the missing transverse momentum [14], whose magnitude is called missing transverse energy $\left(\mathbb{E}_{\mathrm{T}}\right)$. The identification criteria of each object and additional selections are chosen to reduce all backgrounds other than SM $t \bar{t}$ production.

Electron candidates are reconstructed from clusters of energy deposits in the electromagnetic calorimeter, which are then matched to hits in the silicon tracker. Electron identification is based on shower-shape and track-cluster matching variables [15]. Electrons are required to have $p_{\mathrm{T}}>20 \mathrm{GeV}$ and $|\eta|<2.5$ and are excluded if they are in the transition region between the barrel and endcap calorimeters, $1.4442<|\eta|<1.5560$, because their reconstruction in this region is degraded due to additional material there. The electron track must pass within $0.04 \mathrm{~cm}$ of the primary vertex in the plane transverse to the beam. Additionally, electrons coming from photon conversions in the detector material are rejected if there are missing hits in the inner tracker layers or if there is another close track with opposite charge and with a similar polar angle.

Muons are reconstructed using the information from the muon detectors and the silicon tracker [16]. The reconstructed muon track must be within $0.02 \mathrm{~cm}$ of the primary vertex in the plane transverse to the beam. Muons are required to have $p_{\mathrm{T}}>20 \mathrm{GeV}$ and $|\eta|<2.4$.

To remove leptons arising from decays of hadrons immersed in jets, the electrons and muons are required to be isolated. The isolation requirement is based on the ratio of the total transverse energy observed from all particles in a cone of size $R=\sqrt{(\Delta \phi)^{2}+(\Delta \eta)^{2}}<0.3$ centered on the direction of the lepton to the transverse momentum of the lepton. This quantity must be less than 0.17 for electrons and less than 0.20 for muons.

In order to reduce the background from low-mass dilepton resonances, events are rejected if the dilepton invariant mass $M_{\ell \ell}<12 \mathrm{GeV}$. To suppress the contribution from $Z$-boson production, a veto on events with $76<M_{\ell \ell}<$ $106 \mathrm{GeV}$ is applied in the $e e$ and $\mu \mu$ channels.

Events are required to contain at least two jets, reconstructed using an anti- $k_{\mathrm{T}}$ clustering algorithm with a distance parameter of 0.5 [17]. Corrections are applied to account for the dependence of the detector response to jets as a function of $\eta$ and $p_{\mathrm{T}}$ and the effect of pileup (multiple $p p$ collisions) [18]. The corrections are based on in-situ calibration using dijet and $\gamma / Z+$ jet samples. In the $p_{\mathrm{T}}$ region above $\sim 1 \mathrm{TeV}$, where the statistics of the calibration samples become insufficient, the jet energy scale is constrained using the single particle response from test beam data [18]. All corrections are propagated to recalculate the missing transverse energy. The jets are required to have $p_{\mathrm{T}}>30 \mathrm{GeV}$ and $|\eta|<2.5$. Additionally, at least one of the jets is required to be tagged as a $b$ jet based on the identification of a secondary vertex [19].

Finally, in order to further reduce the DY and multijet backgrounds in the $e e$ channel, and the DY contribution in the $\mu \mu$ channel, a requirement of $\mathscr{E}_{\mathrm{T}}>30 \mathrm{GeV}$ is applied in these channels. The DY and multijet backgrounds are negligible in the $e \mu$ channel.

\section{SIGNAL AND BACKGROUND MODELING}

The signal efficiency and background rejection of the selection outlined above are determined from simulation studies augmented where necessary by corrections based on control samples in data. The resonance signal $Z^{\prime} \rightarrow t \bar{t}$ is modeled using the MADGRAPH 5.1.1 [20] Monte Carlo (MC) event generator, with the top quark mass $\left(M_{t}\right)$ set to 172.5 GeV and CTEQ6L [21] parton distribution functions. Samples are generated with resonance masses between 750 and $3000 \mathrm{GeV}$, and for two resonance-width scenarios: narrow $\left(\Gamma_{Z^{\prime}}=0.012 M_{Z^{\prime}}\right)$ and wide $\left(\Gamma_{Z^{\prime}}=0.10 M_{Z^{\prime}}\right)$. To calculate the expected number of signal events, we use cross sections for a leptophobic topcolor $Z^{\prime}$ [22]. A scale factor of 1.3 is used to account for next-to-leading-order (NLO) corrections [23].

The background events from SM $t \bar{t}, \mathrm{DY}$, and $W \rightarrow \ell \nu$ are generated using MADGRAPH 5.1.1. Diboson events are generated using PYTHIA 6.424 [24], and single-top quark events are generated using POWHEG 1.0 r1380 [25-27]. The same set of parton distribution functions are used for each process as for the resonance signal $Z^{\prime}$. The MADGRAPH and POWHEG events are processed through PYTHIA in order to add the initial- and final-state radiation and showering, together with the production of the underlying event [28]. To estimate the expected number of background events, the background samples are normalized to the theoretical cross sections shown in Table I. All MC events are processed through a simulation of the CMS detector based on GEANT4 [34] and are overlaid with events from minimum-bias interaction to account for pileup effects at high instantaneous

TABLE I. Theoretical cross sections, including higher-order corrections, for the SM backgrounds [29-33].

\begin{tabular}{lc}
\hline \hline Background & Cross section $[\mathrm{pb}]$ \\
\hline$t \bar{t}$ & 160 \\
$\mathrm{DY}\left(M_{\ell \ell}>10 \mathrm{GeV}\right)$ & 15000 \\
$W W \rightarrow 2 \ell 2 \nu$ & 4.5 \\
$W Z \rightarrow 3 \ell \nu$ & 0.61 \\
$Z Z$ (inclusive) & 7.4 \\
$W \rightarrow \ell \nu$ & 31000 \\
Single top & 85 \\
\hline \hline
\end{tabular}



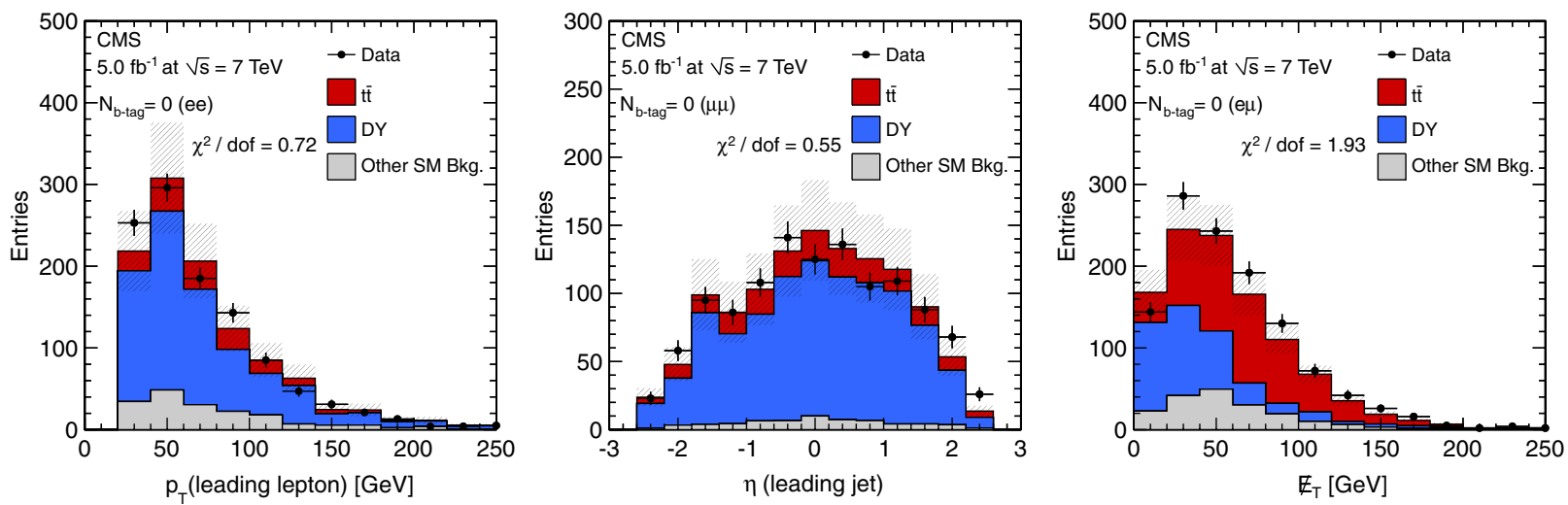

FIG. 1 (color online). Distributions of the transverse momentum of the highest- $p_{\mathrm{T}}$ electron in the $e e$ channel, the pseudorapidity of the highest- $p_{\mathrm{T}}$ jet in the $\mu \mu$ channel, and the missing transverse energy in the $e \mu$ channel in the control sample (zero $b$-tagged jets). The hatched region indicates systematic uncertainties on the sum of SM backgrounds discussed in Sec. VII.

luminosity. An additional set of corrections is applied to account for data-MC differences. These include reweighting $\mathrm{MC}$ events to match the overlaid pileup distribution to that inferred from the data and applying a scale factor of 0.95 per tagged $b$ jet [19] to account for the observed difference in $b$-tagging efficiency between data and MC. The $b$-tagging efficiency is measured using $t \bar{t}$ and muon + jets events [19], with the uncertainty on the data/MC scale factor amounting to $10 \%-20 \%$.

The simulation of the DY background does not adequately reproduce the production rate, especially in the presence of missing transverse energy. The overall normalization for the DY process is therefore obtained using data in the DY-enriched region of $76<M_{\ell \ell}<106 \mathrm{GeV}$ in the $e e$ and $\mu \mu$ channels. This region is excluded from the data set used for the main analysis and thus provides an independent DY control sample. In the $e \mu$ channel, the DYenriched region is between $40<M_{\ell \ell}<70 \mathrm{GeV}$ since the main contribution to DY in this channel is from $Z / \gamma^{*} \rightarrow \tau \bar{\tau}$ events. These events have a peak at lower dilepton masses on account of the invisible decay products of the $\tau$ lepton. As the $Z$-mass veto is not applied in the $e \mu$ channel, the requirement of at least two jets is modified to exactly one jet in order to ensure the exclusion of this calibration sample from the main signal sample. The normalization factors obtained are $1.34 \pm 0.03,1.24 \pm 0.02$, and $1.20 \pm$ 0.05 in the $e e, \mu \mu$, and $e \mu$ channels, respectively.

The multijet background is estimated directly from data. This background is from misidentified leptons or genuine leptons from semileptonic decays of $b / \bar{b}$ or $c / \bar{c}$ quarks, which pass the isolation requirement. It is determined from data by inverting the isolation criteria for both leptons, and then extrapolating that yield to the signal region. The extrapolation is performed by multiplying the yield by a normalization factor that accounts for the isolation efficiency obtained from like-sign lepton events, defined as

$$
f_{\mathrm{QCD}}=\frac{N_{\mathrm{data}}^{ \pm \pm \text {isolated }}-N_{\mathrm{MC}}^{ \pm \pm \text {,isolated }}}{N_{\text {data }}^{ \pm \pm, \text {non-isolated }}}
$$

where $N^{ \pm \pm}$represents the number of like-sign lepton events with the isolation criteria on both leptons either applied or inverted, and MC represents background predictions for $t \bar{t}$, DY, diboson, $W \rightarrow \ell \nu$, and single-top quark events. The multijet estimate was cross-checked with an alternate method in a similar analysis with the same final state, and good agreement was observed [35].

In order to check the background modeling, a control sample is created by requiring zero $b$-tagged jets. This sample is dominated by non- $t \bar{t}$ backgrounds. Figure 1 shows representative distributions from this sample in the $e e, \mu \mu$, and $e \mu$ channels. The shape of the multijet background distribution is derived directly from the data using a control sample of nonisolated leptons, in contrast to the other background shapes, which are taken from simulation. Good agreement is observed between data and background prediction in all three channels.

\section{EVENT YIELDS}

The number of events for the expected SM backgrounds and the observed data after all selections and corrections to account for data-MC differences are listed in Table II. The uncertainties shown for the backgrounds are from the systematic effects discussed in Sec. VII. There is good agreement between data and SM backgrounds in all three

TABLE II. Event yields for SM backgrounds and data in the $e e, \mu \mu$, and $e \mu$ channels.

\begin{tabular}{lccc}
\hline \hline Sample & $e e$ & $\mu \mu$ & $e \mu$ \\
\hline$t \bar{t}$ & $2210 \pm 460$ & $2550 \pm 550$ & $7300 \pm 1500$ \\
DY & $410 \pm 130$ & $420 \pm 130$ & $179 \pm 57$ \\
Diboson & $11.5 \pm 1.5$ & $15.4 \pm 2.0$ & $32.3 \pm 4.2$ \\
$W \rightarrow \ell \nu$ & $17_{-17}^{+24}$ & $0_{-0}^{+3.4}$ & $26_{-26}^{+37}$ \\
Single top & $106 \pm 15$ & $121 \pm 18$ & $343 \pm 50$ \\
Multijets & $41.8 \pm 6.9$ & $50 \pm 10$ & $103 \pm 14$ \\
Total background & $2790 \pm 510$ & $3150 \pm 600$ & $8000 \pm 1500$ \\
Data & 2690 & 3098 & 7704 \\
\hline \hline
\end{tabular}


TABLE III. Event yields for a leptophobic $Z^{\prime}$ in $e e, \mu \mu$, and $e \mu$ channels.

\begin{tabular}{lccc}
\hline \hline Sample & $e e$ & $\mu \mu$ & $e \mu$ \\
\hline & $\Gamma_{Z^{\prime}} / M_{Z^{\prime}}=0.012$ & & \\
$Z^{\prime}(750 \mathrm{GeV})$ & 67 & 79 & 200 \\
$Z^{\prime}(1000 \mathrm{GeV})$ & 26 & 28 & 68 \\
$Z^{\prime}(1250 \mathrm{GeV})$ & 8.2 & 9.8 & 22 \\
$Z^{\prime}(1500 \mathrm{GeV})$ & 2.9 & 3.1 & 7.0 \\
$Z^{\prime}(2000 \mathrm{GeV})$ & 0.3 & 0.4 & 0.8 \\
& $\Gamma_{Z^{\prime}} / M_{Z^{\prime}}=0.10$ & & \\
$Z^{\prime}(1000 \mathrm{GeV})$ & 180 & 200 & 480 \\
$Z^{\prime}(1500 \mathrm{GeV})$ & 23 & 26 & 57 \\
$Z^{\prime}(2000 \mathrm{GeV})$ & 2.9 & 2.9 & 7.0 \\
$Z^{\prime}(3000 \mathrm{GeV})$ & 0.1 & 0.1 & 0.2 \\
\hline \hline
\end{tabular}

channels. For comparison, the numbers of expected $Z^{\prime}$ signal events for $M_{Z^{\prime}}=750-3000 \mathrm{GeV}$ are listed in Table III.

Distributions of the transverse momentum of the highest- $p_{\mathrm{T}}$ electron in the $e e$ channel, the pseudorapidity of the highest- $p_{\mathrm{T}}$ jet in the $\mu \mu$ channel, and $\Delta \phi$ between the missing transverse momentum and highest- $p_{\mathrm{T}}$ lepton in the $e \mu$ channel are shown in Fig. 2. Also shown are the distributions of the missing transverse energy in the three channels. There is good agreement between data and the sum of all SM backgrounds. Similarly, a good agreement is seen in all four-vector distributions $\left(p_{\mathrm{T}}, \eta\right.$, and $\left.\phi\right)$ of all final-state objects in the three channels.

The $t \bar{t}$ invariant mass is constructed using the fourvectors of the two leading leptons, the two leading jets, and the missing transverse energy. The longitudinal momenta $p_{z}$ of the two neutrinos in the final state cannot be measured experimentally and are set to zero. The $t \bar{t}$ invariant mass distributions for the data, the sum of all backgrounds, and the $Z^{\prime}$ signals for the narrow-width scenario $\left(\Gamma_{Z^{\prime}}=0.012 M_{Z^{\prime}}\right)$ are shown in Fig. 3 for the $e e, \mu \mu$, and $e \mu$ channels. The data are described well by the SM backgrounds, and there is no statistically significant evidence for the presence of a $Z^{\prime}$ signal.

\section{BAYESIAN NEURAL NETWORK ANALYSIS}

A multivariate analysis, based on Bayesian neural networks (BNN) [36], has been carried out to provide a more powerful discriminant between backgrounds and the $Z^{\prime}$ signal than that based on invariant mass alone. The discriminant lies in the interval $[0,1]$. It is constructed such that the signal events tend to have a value closer to 1 than the background events that peak instead closer to 0 . To build this discriminant the background is defined as the
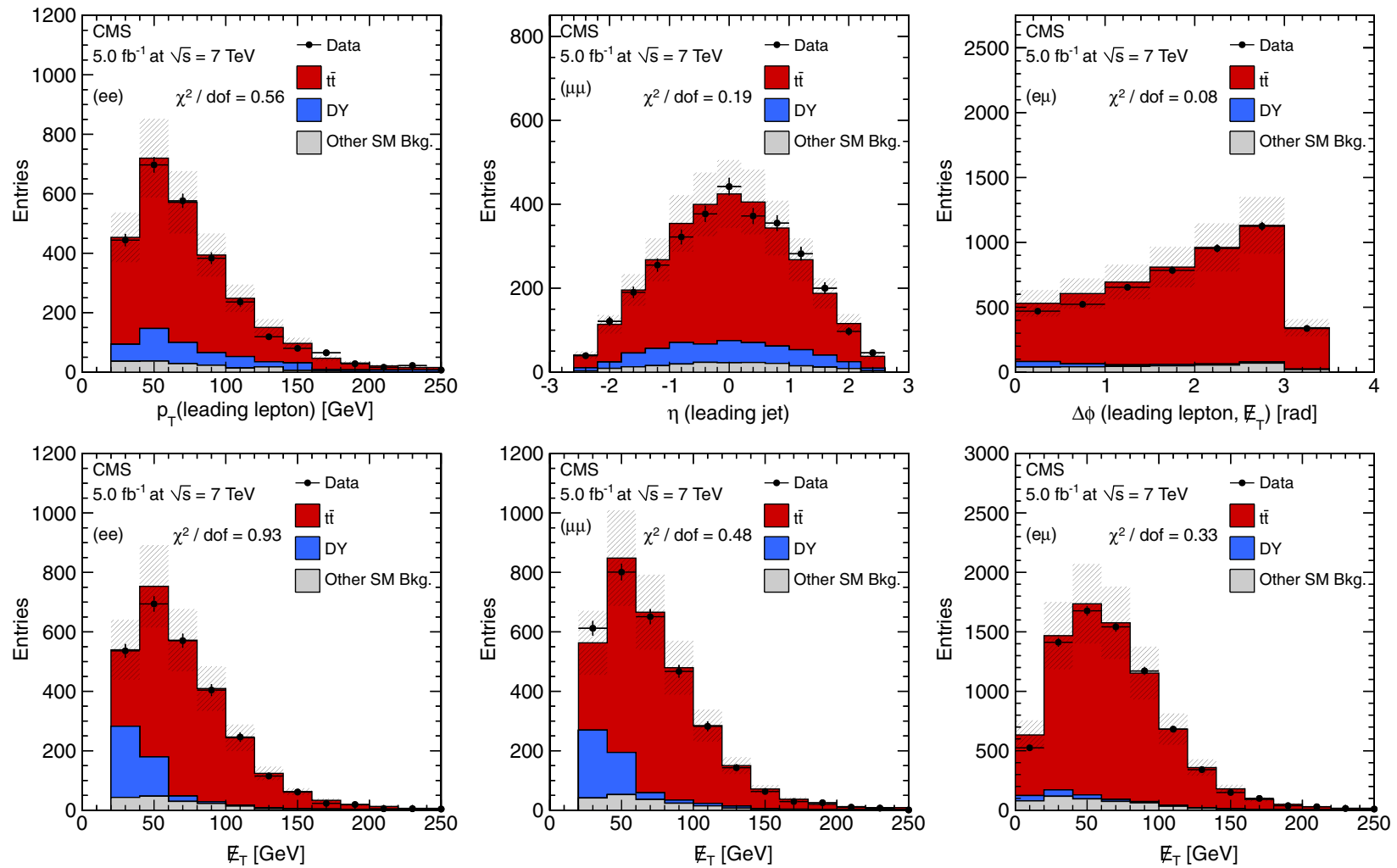

FIG. 2 (color online). Distributions of the transverse momentum of the highest- $p_{\mathrm{T}}$ electron in the $e e$ channel, the pseudorapidity of the highest- $p_{\mathrm{T}}$ jet in the $\mu \mu$ channel, and $\Delta \phi$ between the missing transverse momentum and highest- $p_{\mathrm{T}}$ lepton in the $e \mu$ channel (top row), and the missing transverse energy in the three channels (bottom row). The hatched region indicates systematic uncertainties on the sum of SM backgrounds. 
SEARCH FOR $Z^{\prime}$ RESONANCES DECAYING TO ...

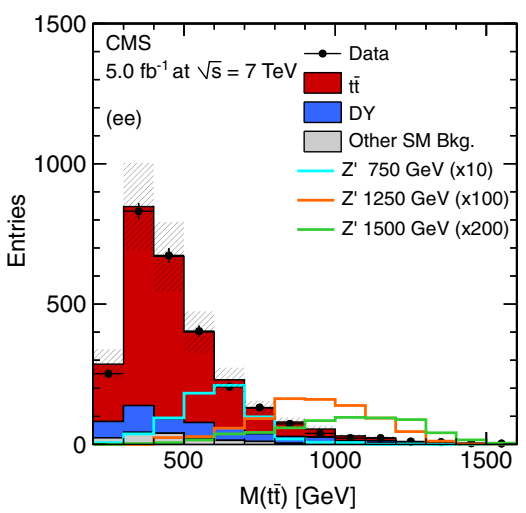

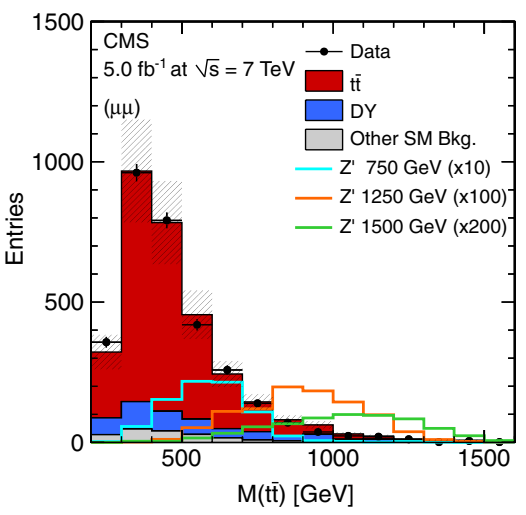

PHYSICAL REVIEW D 87, 072002 (2013)

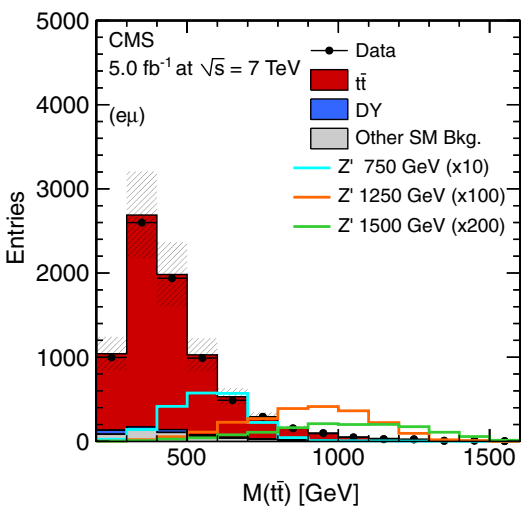

FIG. 3 (color online). Distributions of the $t \bar{t}$ invariant mass for the $e e, \mu \mu$, and $e \mu$ channels. The $p_{z}$ values for both neutrinos are set to zero. The hatched region indicates systematic uncertainties on the sum of SM backgrounds. The $Z^{\prime}$ signal corresponds to a resonance width of $\Gamma_{Z^{\prime}}=0.012 M_{Z^{\prime}}$ and has been scaled up so as to be visible.

sum of all SM processes, and the signal is set to $M_{Z^{\prime}}=$ $750 \mathrm{GeV}$ for narrow width, and $M_{Z^{\prime}}=1000 \mathrm{GeV}$ for wide width. The separation power increases with hypothetical $Z^{\prime}$ mass. Thus, using the $Z^{\prime}$ simulated sample with the lowest $Z^{\prime}$ mass for the signal when training the BNN ensures good discrimination between signal and background even for the higher $Z^{\prime}$ masses. A separate discriminant is constructed in each of the three channels. As inputs to the training, the following 17 variables are used in each of the two $Z^{\prime}$-width scenarios, and in each channel:
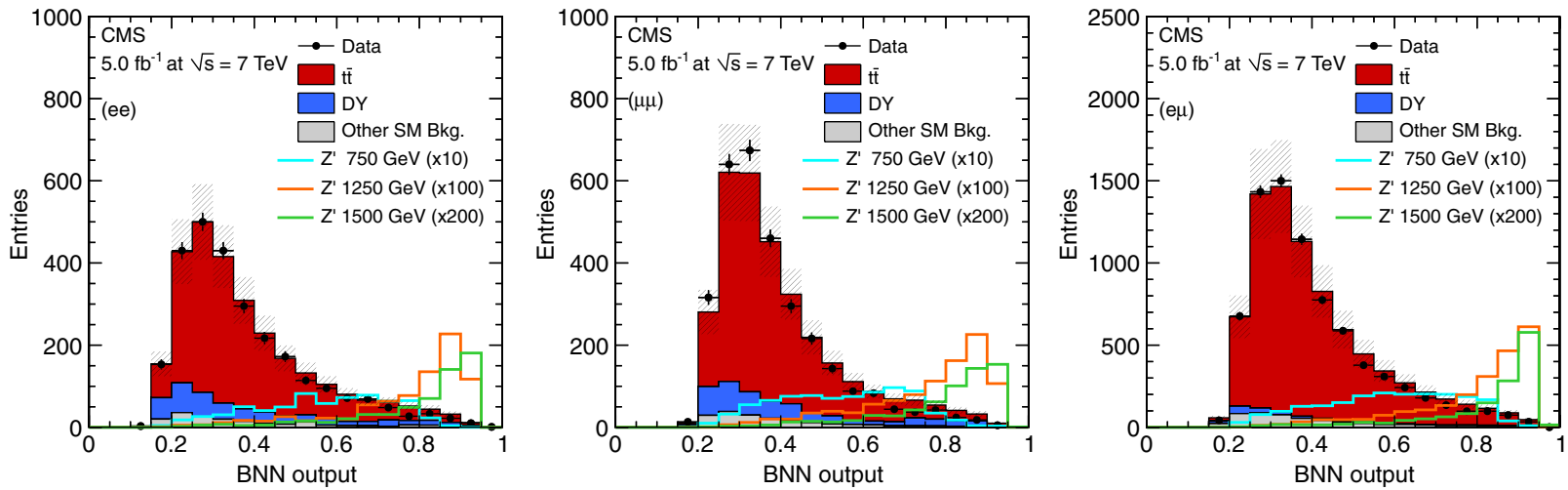

FIG. 4 (color online). Distributions of the BNN output discriminant for the $e e, \mu \mu$, and $e \mu$ channels. The hatched region indicates systematic uncertainties on the sum of SM backgrounds. The $Z^{\prime}$ signal corresponds to a resonance width of $\Gamma_{Z^{\prime}}=0.012 M_{Z^{\prime}}$ and has been scaled up so as to be visible.
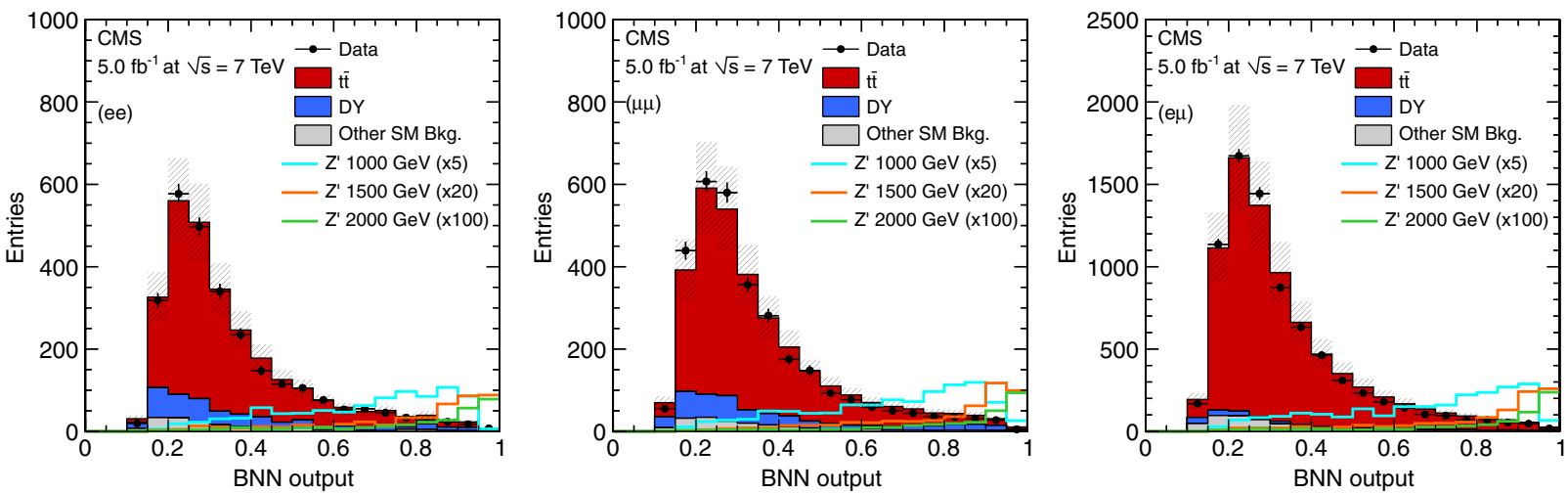

FIG. 5 (color online). Distributions of the BNN output discriminant for the $e e, \mu \mu$, and $e \mu$ channels. The hatched region indicates systematic uncertainties on the sum of SM backgrounds. The $Z^{\prime}$ signal corresponds to a resonance width of $\Gamma_{Z^{\prime}}=0.10 M_{Z^{\prime}}$ and has been scaled up so as to be visible. 
(i) $p_{\mathrm{T}}$ and $\eta$ of the highest- $p_{\mathrm{T}}$ lepton,

(ii) $p_{\mathrm{T}}, \eta$, and $\Delta \phi$ of the second highest- $p_{\mathrm{T}}$ lepton,

(iii) $p_{\mathrm{T}}, \eta$, and $\Delta \phi$ of the highest- $p_{\mathrm{T}}$ jet,

(iv) $p_{\mathrm{T}}, \eta$, and $\Delta \phi$ of the second highest- $p_{\mathrm{T}}$ jet,

(v) $\mathbb{E}_{\mathrm{T}}$, and $\Delta \phi$ of the missing transverse momentum,

(vi) $p_{\mathrm{T}}, \eta$, and $\Delta \phi$ of the highest- $p_{\mathrm{T}} b$-tagged jet, and number $n_{b}$ of $b$-tagged jets,

where $\Delta \phi$ is the difference in azimuth between the object and the highest- $p_{\mathrm{T}}$ lepton. All input variables are internally transformed by the BNN to have a range of $[-1,1]$. This set of input variables constitutes the full array of fourvectors of final-state objects that are measured in the analysis, along with additional information about the $b$-tagged jets. Using additional derived quantities such as the reconstructed $t \bar{t}$ invariant mass as an input to the BNN does not improve the performance of the BNN.

The resulting BNN outputs for the observed data, the SM background, and the $Z^{\prime}$ signals for $\Gamma_{Z^{\prime}}=0.012 M_{Z^{\prime}}$ and $\Gamma_{Z^{\prime}}=0.10 M_{Z^{\prime}}$ are shown in Figs. 4 and 5, respectively, for the $e e, \mu \mu$, and $e \mu$ channels. There is good agreement between data and the SM background in all three channels with no evidence of a resonance signal. Upper limits are set on the production cross section of $Z^{\prime}$ times its branching fraction to top quark-antiquark pairs $\left[\sigma_{Z^{\prime}} \mathcal{B}\left(Z^{\prime} \rightarrow t \bar{t}\right)\right]$.

\section{SYSTEMATIC UNCERTAINTIES}

The signal and background models are affected by a number of systematic uncertainties, which are propagated into the limit calculation. The uncertainties are divided into two categories: those that affect only the overall normalization of a process ("rate") and those that affect also the distribution of the BNN discriminant ("shape"). The rate effects include the uncertainty on predicted cross section and normalization for each SM background based on data, as discussed in Sec. IV, and the uncertainties from integrated luminosity, lepton identification and isolation, $b$-tagging scale factor, jet energy scale, and pileup reweighting for both SM background and $Z^{\prime}$ signals. Rate uncertainties are also included for the SM $t \bar{t}$ and $W \rightarrow \ell \nu$ events from variations in the renormalization and factorization scales $(\lambda)$ and the matching scale for jet production threshold between jets from matrix-element generation in MADGRAPH and parton showering in PYTHIA [37]. The nominal value of $\lambda$ is set to a dynamical mass scale of $\left(2 M_{t}\right)^{2}+\left(\sum p_{\mathrm{T}}^{\text {jet }}\right)^{2}$ for SM $t \bar{t}$ events, and $\left(M_{W}\right)^{2}+$ $\left(\sum p_{\mathrm{T}}^{\text {jet }}\right)^{2}$ with $M_{W}=80.4 \mathrm{GeV}$ for $W \rightarrow \ell \nu$ events. The nominal value of matching scale is set to $20 \mathrm{GeV}$. The $\lambda$ and matching scales are each varied up and down by a factor of 2 with respect to their nominal values in order to estimate the uncertainty. The shape effects include the change in shape of the BNN distributions from the uncertainties from jet energy scale, pileup reweighting, and $\lambda$ and matching scales. The uncertainty due to parton distribution functions is negligible and therefore not included. The uncertainty on the multijet background is dominated
TABLE IV. Rate (top part) and shape (bottom part) uncertainties, from different components, affecting the total expected number of signal and background events. The uncertainties on the $\lambda$ and matching scales in SM $t \bar{t}$, the jet energy scale, and the pileup reweighting are indicated by their range across bins of the BNN distribution.

\begin{tabular}{lc}
\hline \hline Component & Uncertainty [\%] \\
\hline$t \bar{t}$ cross section & 15 \\
DY normalization & 30 \\
Diboson cross section & 3.8 \\
$W \rightarrow \ell \nu$ cross section & 5.0 \\
Single top cross section & 7.7 \\
QCD normalization & $13(e e), 18.2(\mu \mu), 9.7(e \mu)$ \\
Integrated luminosity & 2.2 \\
Lepton identification & 2.0 \\
$b$-tagging scale factor & 10 \\
$\lambda$ scale $(W \rightarrow \ell \nu)$ & 100 \\
Matching scale $(W \rightarrow \ell \nu)$ & 100 \\
$\lambda$ scale $(t \bar{t})$ & $1.9-2.9$ \\
Matching scale $(t \bar{t})$ & $3.4-5.5$ \\
Jet energy scale & $0.3-4.7$ \\
Pileup reweighting & $0.3-2.4$ \\
\hline \hline
\end{tabular}

by the statistical uncertainty of the same-sign samples in Eq. (1). All uncertainties are summarized in Table IV, where each row represents an independent entity. The dominant source of systematic uncertainty in the background estimate is due to the $t \bar{t}$ cross section uncertainty of $15 \%$ which is fully correlated between the channels. The total uncertainty on the sum of all SM backgrounds is $18 \%$.

\section{RESULTS}

With no excess observed, upper limits on $\sigma_{Z^{\prime}} \mathcal{B}\left(Z^{\prime} \rightarrow t \bar{t}\right)$ at the $95 \%$ confidence level (C.L.) for different values of $M_{Z^{\prime}}$ are set using the $\mathrm{CL}_{s}$ criteria [38,39]. All systematic effects are included with correlations across the different samples and channels. The sensitivity of the results is estimated using the invariant mass distributions shown in Fig. 3 and the BNN output distributions shown in Fig. 4 and comparing the expected limit on $\sigma_{Z^{\prime}} \mathcal{B}\left(Z^{\prime} \rightarrow t \bar{t}\right)$ for the two methods at $M_{Z^{\prime}}=750 \mathrm{GeV}$ and $\Gamma_{Z^{\prime}}=0.012 M_{Z^{\prime}}$. An expected limit is obtained from an ensemble of simulated pseudodata sets, where each set is constructed from the background-only hypothesis. Using the BNN distribution improves the expected limit by $29 \%$ compared to using invariant mass distribution. For this reason, the more sensitive BNN technique is used for the subsequent measurements. The resulting expected limits and the observed limits using data are shown in Fig. 6 for both narrow and wide resonances.

The theoretical predictions for a leptophobic $Z^{\prime}[22,23]$ are used to exclude heavy $Z^{\prime}$ resonances of masses $M_{Z^{\prime}}<$ $1.3 \mathrm{TeV}$ for a width $\Gamma_{Z^{\prime}}=0.012 M_{Z^{\prime}}$, and $M<1.9 \mathrm{TeV}$ for a width $\Gamma_{Z^{\prime}}=0.10 M_{Z^{\prime}}$. In the current analysis, the expected lower limits on $M_{Z^{\prime}}$ are $1.1 \mathrm{TeV}$ and $1.7 \mathrm{TeV}$ for $\Gamma_{Z^{\prime}}=0.012 M_{Z^{\prime}}$ and $\Gamma_{Z^{\prime}}=0.10 M_{Z^{\prime}}$, respectively. 

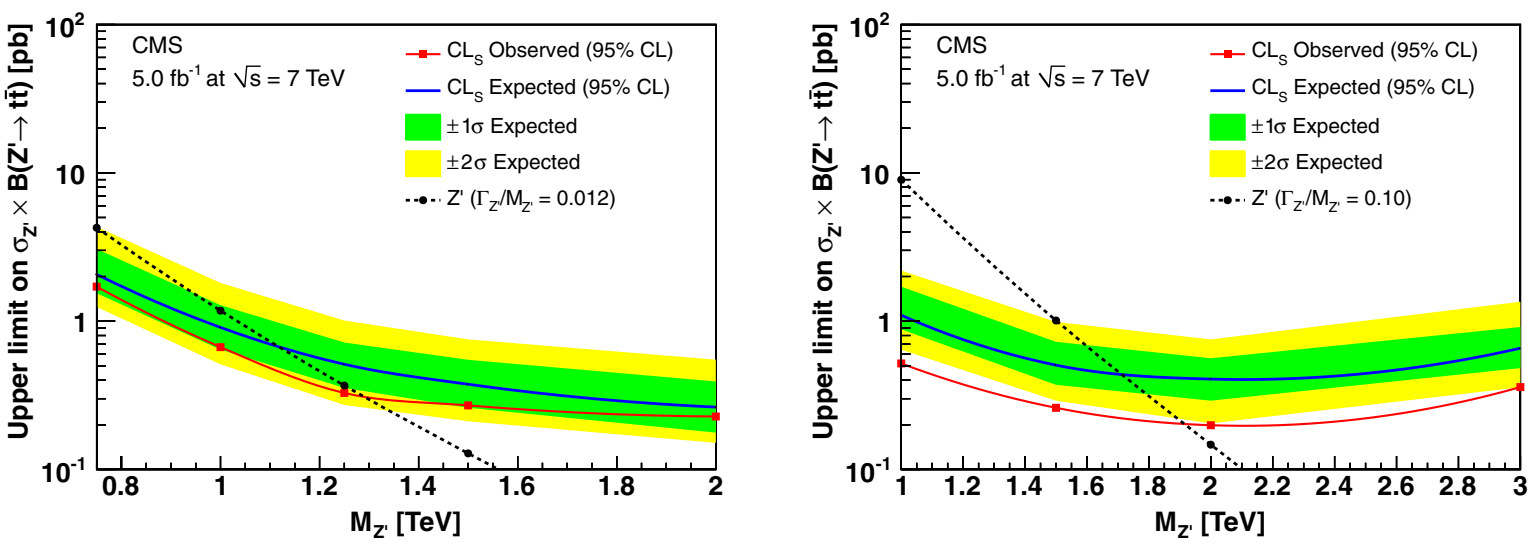

FIG. 6 (color online). Upper limits on $\sigma_{Z^{\prime}} \mathcal{B}\left(Z^{\prime} \rightarrow t \bar{t}\right)$ versus $M_{Z^{\prime}}$ for narrow and wide resonances. Also shown is the theoretical prediction for the $Z^{\prime}$.

\section{SUMMARY}

A data sample, corresponding to an integrated luminosity of $5.0 \mathrm{fb}^{-1}$ collected in $p p$ collisions at $\sqrt{s}=7 \mathrm{TeV}$, has been analyzed in a search for heavy resonances decaying to top quark-antiquark pairs with subsequent leptonic decay of both top quark and antiquark. No excess beyond the standard model prediction is observed. Upper limits at the $95 \%$ C.L. are derived on the product of the production cross section and branching fraction for these decays, for various masses of narrow and wide resonances. The existence of a leptophobic $Z^{\prime}$ topcolor particle is excluded for $M_{Z^{\prime}}<1.3 \mathrm{TeV}$ with $\Gamma_{Z^{\prime}}=0.012 M_{Z^{\prime}}$, and for $M_{Z^{\prime}}<$ $1.9 \mathrm{TeV}$ with $\Gamma_{Z^{\prime}}=0.10 M_{Z^{\prime}}$.

\section{ACKNOWLEDGMENTS}

We congratulate our colleagues in the CERN accelerator departments for the excellent performance of the LHC and thank the technical and administrative staffs at CERN and at other CMS institutes for their contributions to the success of the CMS effort. In addition, we gratefully acknowledge the computing centers and personnel of the Worldwide LHC Computing Grid for delivering so effectively the computing infrastructure essential to our analyses. Finally, we acknowledge the enduring support for the construction and operation of the LHC and the CMS detector provided by the following funding agencies: BMWF and FWF (Austria); FNRS and FWO (Belgium); CNPq, CAPES, FAPERJ, and FAPESP (Brazil); MEYS (Bulgaria); CERN; CAS, MoST, and NSFC (China); COLCIENCIAS (Colombia); MSES (Croatia); RPF (Cyprus); MoER, SF0690030s09 and ERDF (Estonia); Academy of Finland, MEC, and HIP (Finland); CEA and CNRS/ IN2P3 (France); BMBF, DFG, and HGF (Germany); GSRT (Greece); OTKA and NKTH (Hungary); DAE and DST (India); IPM (Iran); SFI (Ireland); INFN (Italy); NRF and WCU (Korea); LAS (Lithuania); CINVESTAV, CONACYT, SEP, and UASLP-FAI (Mexico); MSI (New Zealand); PAEC (Pakistan); MSHE and NSC (Poland); FCT (Portugal); JINR (Armenia, Belarus, Georgia, Ukraine, Uzbekistan); MON, RosAtom, RAS, and RFBR (Russia); MSTD (Serbia); SEIDI and CPAN (Spain); Swiss Funding Agencies (Switzerland); NSC (Taipei); ThEP, IPST, and NECTEC (Thailand); TUBITAK and TAEK (Turkey); NASU (Ukraine); STFC (United Kingdom); DOE and NSF (USA).
[1] ATLAS Collaboration, Phys. Lett. B 716, 1 (2012).

[2] CMS Collaboration, Phys. Lett. B 716, 30 (2012).

[3] C. T. Hill, Phys. Lett. B 266, 419 (1991).

[4] S. Weinberg, Phys. Rev. D 13, 974 (1976).

[5] C. T. Hill, Phys. Lett. B 345, 483 (1995); updates in arXiv: hep-ph/9911288.

[6] T. Aaltonen et al. (CDF Collaboration), Phys. Rev. D 84, 072004 (2011).

[7] V. M. Abazov et al. (D0 Collaboration), Phys. Rev. D 85, 051101 (2012).

[8] ATLAS Collaboration, Eur. Phys. J. C 72, 2083 (2012).

[9] CMS Collaboration, J. High Energy Phys. 09 (2012) 029.
[10] CMS Collaboration, J. High Energy Phys. 12 (2012) 015.

[11] CMS Collaboration, CMS Physics Analysis Summary Report No. CMS-PAS-SMP-12-008, 2012, http://cds.cern .ch/record/1434360.

[12] CMS Collaboration, JINST 3, S08004 (2008).

[13] CMS Collaboration, CMS Physics Analysis Summary Report No. CMS-PAS-PFT-10-002, 2010, http://cds.cern .ch/record/1279341.

[14] CMS Collaboration, JINST 6, P09001 (2011).

[15] CMS Collaboration, CMS Physics Analysis Summary Report No. CMS-PAS-EGM-10-004, 2010, http://cds.cern .ch/record/1299116. 
[16] CMS Collaboration, JINST 7, P10002 (2012).

[17] M. Cacciari, G. P. Salam, and G. Soyez, J. High Energy Phys. 04 (2008) 063.

[18] CMS Collaboration, JINST 6, P11002 (2011).

[19] CMS Collaboration, CMS Physics Analysis Summary Report No. CMS-PAS-BTV-11-004, 2011, http://cds.cern .ch/record/1427247.

[20] J. Alwall, M. Herquet, F. Maltoni, O. Mattelaer, and T. Stelzer, J. High Energy Phys. 06 (2011) 128.

[21] J. Pumplin, D. R. Stump, J. Huston, H. L. Lai, P. Nadolsky, and W. K. Tung, J. High Energy Phys. 07 (2002) 012.

[22] R. M. Harris and S. Jain, Eur. Phys. J. C 72, 2072 (2012).

[23] J. Gao, C. S. Li, B. H. Li, H. X. Zhu, and C.-P. Yuan, Phys. Rev. D 82, 014020 (2010).

[24] T. Sjöstrand, S. Mrenna, and P. Skands, J. High Energy Phys. 05 (2006) 026.

[25] S. Alioli, P. Nason, C. Oleari, and E. Re, J. High Energy Phys. 09 (2009) 111.

[26] S. Alioli, P. Nason, C. Oleari, and E. Re, J. High Energy Phys. 06 (2010) 043.
[27] E. Re, Eur. Phys. J. C 71, 1547 (2011).

[28] R. Field, arXiv:1010.3558.

[29] J. M. Campbell and R. K. Ellis, Nucl. Phys. B, Proc. Suppl. 205-206, 10 (2010).

[30] K. Melnikov and F. Petriello, Phys. Rev. D 74, 114017 (2006).

[31] N. Kidonakis, Phys. Rev. D 83, 091503 (2011).

[32] N. Kidonakis, Phys. Rev. D 81, 054028 (2010).

[33] N. Kidonakis, Phys. Rev. D 82, 054018 (2010).

[34] S. Agostinelli et al., Nucl. Instrum. Methods Phys. Res., Sect. A 506, 250 (2003).

[35] CMS Collaboration, arXiv:1211.2220.

[36] R. M. Neal, Bayesian Learning for Neural Networks (Springer-Verlag, New York, 1996); we used the software for Flexible Bayesian Modeling from http://www.cs .toronto.edu/ radford/fbm.software.html.

[37] M. L. Mangano, M. Moretti, F. Piccinini, and M. Treccani, J. High Energy Phys. 01 (2007) 013.

[38] A. L. Read, J. Phys. G 28, 2693 (2002).

[39] T. Junk, Nucl. Instrum. Methods Phys. Res., Sect. A 434, 435 (1999).

S. Chatrchyan, ${ }^{1}$ V. Khachatryan, ${ }^{1}$ A. M. Sirunyan, ${ }^{1}$ A. Tumasyan, ${ }^{1}$ W. Adam,${ }^{2}$ E. Aguilo, ${ }^{2}$ T. Bergauer, ${ }^{2}$ M. Dragicevic, ${ }^{2}$ J. Erö, ${ }^{2}$ C. Fabjan, ${ }^{2, b}$ M. Friedl, ${ }^{2}$ R. Frühwirth, ${ }^{2, b}$ V. M. Ghete, ${ }^{2}$ N. Hörmann, ${ }^{2}$ J. Hrubec, ${ }^{2}$ M. Jeitler, ${ }^{2, \mathrm{~b}}$ W. Kiesenhofer, ${ }^{2}$ V. Knünz, ${ }^{2}$ M. Krammer, ${ }^{2, \mathrm{~b}}$ I. Krätschmer, ${ }^{2}$ D. Liko, ${ }^{2}$ I. Mikulec, ${ }^{2}$ M. Pernicka, ${ }^{2, \mathrm{a}}$ D. Rabady, ${ }^{2, \mathrm{c}}$ B. Rahbaran, ${ }^{2}$ C. Rohringer, ${ }^{2}$ H. Rohringer, ${ }^{2}$ R. Schöfbeck, ${ }^{2}$ J. Strauss, ${ }^{2}$ A. Taurok, ${ }^{2}$ W. Waltenberger, ${ }^{2}$ C.-E. Wulz, ${ }^{2, b}$ V. Mossolov, ${ }^{3}$ N. Shumeiko, ${ }^{3}$ J. Suarez Gonzalez, ${ }^{3}$ S. Alderweireldt, ${ }^{4}$ M. Bansal,${ }^{4}$ S. Bansal ${ }^{4}$ T. Cornelis, ${ }^{4}$ E. A. De Wolf, ${ }^{4}$ X. Janssen, ${ }^{4}$ S. Luyckx,${ }^{4}$ L. Mucibello, ${ }^{4}$ S. Ochesanu, ${ }^{4}$ B. Roland, ${ }^{4}$ R. Rougny, ${ }^{4}$ M. Selvaggi, ${ }^{4}$ H. Van Haevermaet, ${ }^{4}$ P. Van Mechelen, ${ }^{4}$ N. Van Remortel, ${ }^{4}$ A. Van Spilbeeck, ${ }^{4}$ F. Blekman, ${ }^{5}$ S. Blyweert, ${ }^{5}$ J. D'Hondt, ${ }^{5}$ R. Gonzalez Suarez, ${ }^{5}$ A. Kalogeropoulos, ${ }^{5}$ M. Maes, ${ }^{5}$ A. Olbrechts, ${ }^{5}$ S. Tavernier, ${ }^{5}$ W. Van Doninck, ${ }^{5}$ P. Van Mulders, ${ }^{5}$ G. P. Van Onsem, ${ }^{5}$ I. Villella, ${ }^{5}$ B. Clerbaux,${ }^{6}$ G. De Lentdecker, ${ }^{6}$ V. Dero, ${ }^{6}$ A. P. R. Gay, ${ }^{6}$ T. Hreus, ${ }^{6}$ A. Léonard, ${ }^{6}$ P.E. Marage, ${ }^{6}$ A. Mohammadi ${ }^{6}$ T. Reis, ${ }^{6}$ L. Thomas, ${ }^{6}$ C. Vander Velde, ${ }^{6}$ P. Vanlaer, ${ }^{6}$ J. Wang, ${ }^{6}$ V. Adler, ${ }^{7}$ K. Beernaert, ${ }^{7}$ A. Cimmino, ${ }^{7}$ S. Costantini, ${ }^{7}$ G. Garcia, ${ }^{7}$ M. Grunewald, ${ }^{7}$ B. Klein, ${ }^{7}$ J. Lellouch, ${ }^{7}$ A. Marinov, ${ }^{7}$ J. Mccartin, ${ }^{7}$ A. A. Ocampo Rios, ${ }^{7}$ D. Ryckbosch, ${ }^{7}$ M. Sigamani, ${ }^{7}$ N. Strobbe,${ }^{7}$ F. Thyssen, ${ }^{7}$ M. Tytgat,${ }^{7}$ S. Walsh, ${ }^{7}$ E. Yazgan, ${ }^{7}$ N. Zaganidis, ${ }^{7}$ S. Basegmez,${ }^{8}$ G. Bruno, ${ }^{8}$ R. Castello, ${ }^{8}$ L. Ceard,${ }^{8}$

C. Delaere, ${ }^{8}$ T. du Pree,${ }^{8}$ D. Favart, ${ }^{8}$ L. Forthomme, ${ }^{8}$ A. Giammanco,,${ }^{8, d}$ J. Hollar, ${ }^{8}$ V. Lemaitre, ${ }^{8}$ J. Liao, ${ }^{8}$

O. Militaru, ${ }^{8}$ C. Nuttens, ${ }^{8}$ D. Pagano, ${ }^{8}$ A. Pin,${ }^{8}$ K. Piotrzkowski,${ }^{8}$ J. M. Vizan Garcia, ${ }^{8}$ N. Beliy, ${ }^{9}$ T. Caebergs, ${ }^{9}$ E. Daubie, ${ }^{9}$ G. H. Hammad, ${ }^{9}$ G. A. Alves, ${ }^{10}$ M. Correa Martins Junior, ${ }^{10}$ T. Martins, ${ }^{10}$ M. E. Pol, ${ }^{10}$ M. H. G. Souza, ${ }^{10}$ W. L. Aldá Júnior, ${ }^{11}$ W. Carvalho, ${ }^{11}$ A. Custódio, ${ }^{11}$ E. M. Da Costa, ${ }^{11}$ D. De Jesus Damiao, ${ }^{11}$

C. De Oliveira Martins, ${ }^{11}$ S. Fonseca De Souza, ${ }^{11}$ H. Malbouisson, ${ }^{11}$ M. Malek, ${ }^{11}$ D. Matos Figueiredo, ${ }^{11}$ L. Mundim, ${ }^{11}$ H. Nogima, ${ }^{11}$ W. L. Prado Da Silva,${ }^{11}$ A. Santoro, ${ }^{11}$ L. Soares Jorge,${ }^{11}$ A. Sznajder, ${ }^{11}$ A. Vilela Pereira, ${ }^{11}$ T. S. Anjos, ${ }^{12 b, e}$ C. A. Bernardes, ${ }^{12 b, e}$ F. A. Dias, ${ }^{12 a, f}$ T. R. Fernandez Perez Tomei, ${ }^{12 a}$ E. M. Gregores ${ }^{12 b, e}$ C. Lagana, ${ }^{12 a}$ F. Marinho, ${ }^{12 a}$ P. G. Mercadante, ${ }^{12 b, e}$ S. F. Novaes, ${ }^{12 a}$ Sandra S. Padula, ${ }^{12 a}$ V. Genchev, ${ }^{13, c}$ P. Iaydjiev, ${ }^{13, c}$ S. Piperov ${ }^{13}$ M. Rodozov,${ }^{13}$ S. Stoykova, ${ }^{13}$ G. Sultanov, ${ }^{13}$ V. Tcholakov, ${ }^{13}$ R. Trayanov, ${ }^{13}$ M. Vutova, ${ }^{13}$ A. Dimitrov,${ }^{14}$ R. Hadjiiska, ${ }^{14}$ V. Kozhuharov,${ }^{14}$ L. Litov, ${ }^{14}$ B. Pavlov, ${ }^{14}$ P. Petkov, ${ }^{14}$ J. G. Bian, ${ }^{15}$ G. M. Chen, ${ }^{15}$ H. S. Chen, ${ }^{15}$ C. H. Jiang, ${ }^{15}$ D. Liang, ${ }^{15}$ S. Liang, ${ }^{15}$ X. Meng, ${ }^{15}$ J. Tao, ${ }^{15}$ J. Wang, ${ }^{15}$ X. Wang, ${ }^{15}$ Z. Wang, ${ }^{15}$ H. Xiao, ${ }^{15}$ M. Xu, ${ }^{15}$ J. Zang, ${ }^{15}$ Z. Zhang, ${ }^{15}$ C. Asawatangtrakuldee, ${ }^{16}$ Y. Ban, ${ }^{16}$ Y. Guo, ${ }^{16}$ W. Li, ${ }^{16}$ S. Liu, ${ }^{16}$ Y. Mao, ${ }^{16}$ S. J. Qian, ${ }^{16}$ H. Teng,,${ }^{16}$ D. Wang, ${ }^{16}$ L. Zhang,,${ }^{16}$ W. Zou, ${ }^{16}$ C. Avila, ${ }^{17}$

C. A. Carrillo Montoya,${ }^{17}$ J. P. Gomez, ${ }^{17}$ B. Gomez Moreno, ${ }^{17}$ A. F. Osorio Oliveros, ${ }^{17}$ J. C. Sanabria, ${ }^{17}$ N. Godinovic, ${ }^{18}$ D. Lelas, ${ }^{18}$ R. Plestina, ${ }^{18, \mathrm{~g}}$ D. Polic, ${ }^{18}$ I. Puljak, ${ }^{18, \mathrm{c}}$ Z. Antunovic, ${ }^{19}$ M. Kovac, ${ }^{19}$ V. Brigljevic,${ }^{20}$ S. Duric,${ }^{20}$ K. Kadija,${ }^{20}$ J. Luetic,${ }^{20}$ D. Mekterovic,${ }^{20}$ S. Morovic, ${ }^{20}$ L. Tikvica, ${ }^{20}$ A. Attikis,${ }^{21}$ M. Galanti, ${ }^{21}$ G. Mavromanolakis, ${ }^{21}$ J. Mousa, ${ }^{21}$ C. Nicolaou ${ }^{21}$ F. Ptochos,${ }^{21}$ P. A. Razis, ${ }^{21}$ M. Finger, ${ }^{22}$ M. Finger, Jr. ${ }^{22}$ Y. Assran, ${ }^{23, \mathrm{~h}}$ S. Elgammal, ${ }^{23, \mathrm{i}}$ A. Ellithi Kamel, ${ }^{23, \mathrm{j}}$ A. M. Kuotb Awad, ${ }^{23, \mathrm{k}}$ M. A. Mahmoud, ${ }^{23, \mathrm{k}}$ A. Radi, ${ }^{23,1, \mathrm{~m}}$ 
M. Kadastik, ${ }^{24}$ M. Müntel,${ }^{24}$ M. Murumaa,${ }^{24}$ M. Raidal, ${ }^{24}$ L. Rebane, ${ }^{24}$ A. Tiko, ${ }^{24}$ P. Eerola, ${ }^{25}$ G. Fedi, ${ }^{25}$ M. Voutilainen, ${ }^{25}$ J. Härkönen, ${ }^{26}$ A. Heikkinen, ${ }^{26}$ V. Karimäki ${ }^{26}$ R. Kinnunen, ${ }^{26}$ M. J. Kortelainen ${ }^{26}$ T. Lampén, ${ }^{26}$

K. Lassila-Perini, ${ }^{26}$ S. Lehti, ${ }^{26}$ T. Lindén, ${ }^{26}$ P. Luukka, ${ }^{26}$ T. Mäenpää, ${ }^{26}$ T. Peltola, ${ }^{26}$ E. Tuominen,,${ }^{26}$

J. Tuominiemi, ${ }^{26}$ E. Tuovinen, ${ }^{26}$ D. Ungaro,${ }^{26}$ L. Wendland, ${ }^{26}$ A. Korpela,${ }^{27}$ T. Tuuva, ${ }^{27}$ M. Besancon, ${ }^{28}$ S. Choudhury, ${ }^{28}$ F. Couderc, ${ }^{28}$ M. Dejardin, ${ }^{28}$ D. Denegri, ${ }^{28}$ B. Fabbro, ${ }^{28}$ J. L. Faure,${ }^{28}$ F. Ferri, ${ }^{28}$ S. Ganjour, ${ }^{28}$

A. Givernaud, ${ }^{28}$ P. Gras, ${ }^{28}$ G. Hamel de Monchenault, ${ }^{28}$ P. Jarry, ${ }^{28}$ E. Locci, ${ }^{28}$ J. Malcles, ${ }^{28}$ L. Millischer, ${ }^{28}$ A. Nayak ${ }^{28}{ }^{28}$. Rander ${ }^{28}$ A. Rosowsky,${ }^{28}$ M. Titov ${ }^{28}$ S. Baffioni, ${ }^{29}$ F. Beaudette, ${ }^{29}$ L. Benhabib,${ }^{29}$ L. Bianchini, ${ }^{29}$ M. Bluj, ${ }^{29, n}$ P. Busson, ${ }^{29}$ C. Charlot, ${ }^{29}$ N. Daci, ${ }^{29}$ T. Dahms, ${ }^{29}$ M. Dalchenko, ${ }^{29}$ L. Dobrzynski, ${ }^{29}$ A. Florent, ${ }^{29}$ R. Granier de Cassagnac, ${ }^{29}$ M. Haguenauer, ${ }^{29}$ P. Miné, ${ }^{29}$ C. Mironov, ${ }^{29}$ I. N. Naranjo, ${ }^{29}$ M. Nguyen, ${ }^{29}$ C. Ochando, ${ }^{29}$ P. Paganini, ${ }^{29}$ D. Sabes,${ }^{29}$ R. Salerno, ${ }^{29}$ Y. Sirois,${ }^{29}$ C. Veelken, ${ }^{29}$ A. Zabi, ${ }^{29}$ J.-L. Agram, ${ }^{30, o}$ J. Andrea,${ }^{30}$ D. Bloch, ${ }^{30}$

D. Bodin, ${ }^{30}$ J.-M. Brom,${ }^{30}$ M. Cardaci, ${ }^{30}$ E. C. Chabert,${ }^{30}$ C. Collard ${ }^{30}$ E. Conte,${ }^{30, o}$ F. Drouhin,,${ }^{30, o}$

J.-C. Fontaine, ${ }^{30, o}$ D. Gelé, ${ }^{30}$ U. Goerlach, ${ }^{30}$ P. Juillot, ${ }^{30}$ A.-C. Le Bihan,${ }^{30}$ P. Van Hove, ${ }^{30}$ S. Beauceron, ${ }^{31}$ N. Beaupere, ${ }^{31}$ O. Bondu, ${ }^{31}$ G. Boudoul,${ }^{31}$ S. Brochet, ${ }^{31}$ J. Chasserat,${ }^{31}$ R. Chierici,${ }^{31, \mathrm{c}}$ D. Contardo, ${ }^{31}$ P. Depasse,${ }^{31}$ H. El Mamouni, ${ }^{31}$ J. Fay, ${ }^{31}$ S. Gascon,${ }^{31}$ M. Gouzevitch, ${ }^{31}$ B. Ille, ${ }^{31}$ T. Kurca,${ }^{31}$ M. Lethuillier, ${ }^{31}$ L. Mirabito, ${ }^{31}$ S. Perries, ${ }^{31}$ L. Sgandurra, ${ }^{31}$ V. Sordini, ${ }^{31}$ Y. Tschudi, ${ }^{31}$ P. Verdier, ${ }^{31}$ S. Viret, ${ }^{31}$ Z. Tsamalaidze, ${ }^{32, p}$ C. Autermann,${ }^{33}$ S. Beranek, ${ }^{33}$ B. Calpas, ${ }^{33}$ M. Edelhoff, ${ }^{33}$ L. Feld, ${ }^{33}$ N. Heracleous, ${ }^{33}$ O. Hindrichs, ${ }^{33}$ R. Jussen, ${ }^{33}$ K. Klein, ${ }^{33}$ J. Merz, ${ }^{33}$ A. Ostapchuk, ${ }^{33}$ A. Perieanu, ${ }^{33}$ F. Raupach, ${ }^{33}$ J. Sammet, ${ }^{33}$ S. Schael, ${ }^{33}$ D. Sprenger, ${ }^{33}$ H. Weber, ${ }^{33}$ B. Wittmer, ${ }^{33}$ V. Zhukov ${ }^{33, q}$ M. Ata ${ }^{34}$ J. Caudron, ${ }^{34}$ E. Dietz-Laursonn, ${ }^{34}$ D. Duchardt, ${ }^{34}$ M. Erdmann,${ }^{34}$ R. Fischer, ${ }^{34}$ A. Güth, ${ }^{34}$ T. Hebbeker, ${ }^{34}$ C. Heidemann, ${ }^{34}$ K. Hoepfner, ${ }^{34}$ D. Klingebiel, ${ }^{34}$ P. Kreuzer,${ }^{34}$ M. Merschmeyer, ${ }^{34}$ A. Meyer, ${ }^{34}$ M. Olschewski, ${ }^{34}$ K. Padeken,${ }^{34}$ P. Papacz, ${ }^{34}$ H. Pieta,${ }^{34}$ H. Reithler,${ }^{34}$ S. A. Schmitz, ${ }^{34}$ L. Sonnenschein, ${ }^{34}$ J. Steggemann, ${ }^{34}$ D. Teyssier, ${ }^{34}$ S. Thüer, ${ }^{34}$ M. Weber, ${ }^{34}$ M. Bontenackels, ${ }^{35}$ V. Cherepanov, ${ }^{35}$ Y. Erdogan, ${ }^{35}$ G. Flügge,${ }^{35}$ H. Geenen, ${ }^{35}$ M. Geisler, ${ }^{35}$ W. Haj Ahmad,${ }^{35}$ F. Hoehle,${ }^{35}$ B. Kargoll, ${ }^{35}$ T. Kress, ${ }^{35}$ Y. Kuessel,,${ }^{35}$ J. Lingemann, ${ }^{35, c}$ A. Nowack, ${ }^{35}$ I. M. Nugent, ${ }^{35}$ L. Perchalla, ${ }^{35}$ O. Pooth,${ }^{35}$ P. Sauerland, ${ }^{35}$ A. Stahl, ${ }^{35}$ M. Aldaya Martin, ${ }^{36}$ I. Asin, ${ }^{36}$ N. Bartosik, ${ }^{36}$ J. Behr, ${ }^{36}$ W. Behrenhoff, ${ }^{36}$ U. Behrens, ${ }^{36}$ M. Bergholz,,${ }^{36, r}$ A. Bethani, ${ }^{36}$ K. Borras, ${ }^{36}$ A. Burgmeier, ${ }^{36}$ A. Cakir, ${ }^{36}$ L. Calligaris, ${ }^{36}$ A. Campbell, ${ }^{36}$ E. Castro, ${ }^{36}$ F. Costanza, ${ }^{36}$ D. Dammann, ${ }^{36}$ C. Diez Pardos, ${ }^{36}$ T. Dorland, ${ }^{36}$ G. Eckerlin, ${ }^{36}$ D. Eckstein, ${ }^{36}$ G. Flucke, ${ }^{36}$ A. Geiser, ${ }^{36}$ I. Glushkov ${ }^{36}$ P. Gunnellini, ${ }^{36}$ S. Habib,${ }^{36}$ J. Hauk, ${ }^{36}$ G. Hellwig,${ }^{36}$ H. Jung, ${ }^{36}$ M. Kasemann,,${ }^{36}$ P. Katsas, ${ }^{36}$ C. Kleinwort, ${ }^{36}$ H. Kluge, ${ }^{36}$ A. Knutsson, ${ }^{36}$ M. Krämer, ${ }^{36}$ D. Krücker, ${ }^{36}$ E. Kuznetsova, ${ }^{36}$ W. Lange, ${ }^{36}$ J. Leonard, ${ }^{36}$ W. Lohmann, ${ }^{36, r}$ B. Lutz,${ }^{36}$ R. Mankel, ${ }^{36}$ I. Marfin, ${ }^{36}$ M. Marienfeld ${ }^{36}$ I.-A. Melzer-Pellmann, ${ }^{36}$ A. B. Meyer, ${ }^{36}$ J. Mnich, ${ }^{36}$ A. Mussgiller, ${ }^{36}$ S. Naumann-Emme, ${ }^{36}$ O. Novgorodova, ${ }^{36}$ F. Nowak, ${ }^{36}$ J. Olzem, ${ }^{36}$ H. Perrey, ${ }^{36}$ A. Petrukhin, ${ }^{36}$ D. Pitzl, ${ }^{36}$ A. Raspereza,${ }^{36}$ P. M. Ribeiro Cipriano, ${ }^{36}$ C. Riedl, ${ }^{36}$ E. Ron, ${ }^{36}$ M. Rosin, ${ }^{36}$ J. Salfeld-Nebgen,${ }^{36}$ R. Schmidt, ${ }^{36, r}$ T. Schoerner-Sadenius, ${ }^{36}$ N. Sen, ${ }^{36}$ A. Spiridonov, ${ }^{36}$ M. Stein,${ }^{36}$ R. Walsh,${ }^{36}$ C. Wissing,,${ }^{36}$ V. Blobel,${ }^{37}$ H. Enderle,${ }^{37}$ J. Erfle, ${ }^{37}$ U. Gebbert, ${ }^{37}$ M. Görner, ${ }^{37}$ M. Gosselink, ${ }^{37}$ J. Haller,${ }^{37}$ T. Hermanns, ${ }^{37}$ R. S. Höing, ${ }^{37}$ K. Kaschube, ${ }^{37}$ G. Kaussen, ${ }^{37}$ H. Kirschenmann, ${ }^{37}$ R. Klanner, ${ }^{37}$ J. Lange, ${ }^{37}$ T. Peiffer, ${ }^{37}$ N. Pietsch, ${ }^{37}$ D. Rathjens, ${ }^{37}$ C. Sander, ${ }^{37}$ H. Schettler, ${ }^{37}$ P. Schleper, ${ }^{37}$ E. Schlieckau, ${ }^{37}$ A. Schmidt, ${ }^{37}$ M. Schröder, ${ }^{37}$ T. Schum, ${ }^{37}$ M. Seidel, ${ }^{37}$ J. Sibille, ${ }^{37, s}$ V. Sola,${ }^{37}$ H. Stadie,${ }^{37}$ G. Steinbrück,${ }^{37}$ J. Thomsen, ${ }^{37}$ L. Vanelderen, ${ }^{37}$ C. Barth, ${ }^{38}$ C. Baus, ${ }^{38}$ J. Berger, ${ }^{38}$ C. Böser, ${ }^{38}$ T. Chwalek,${ }^{38}$ W. De Boer, ${ }^{38}$ A. Descroix,${ }^{38}$ A. Dierlamm, ${ }^{38}$ M. Feindt,${ }^{38}$ M. Guthoff, ${ }^{38, \mathrm{c}}$ C. Hackstein, ${ }^{38}$ F. Hartmann, ${ }^{38, \mathrm{c}}$ T. Hauth, ${ }^{38, \mathrm{c}}$ M. Heinrich, ${ }^{38}$ H. Held, ${ }^{38}$ K. H. Hoffmann, ${ }^{38}$ U. Husemann, ${ }^{38}$ I. Katkov, ${ }^{38, q}$ J. R. Komaragiri, ${ }^{38}$ P. Lobelle Pardo ${ }^{38}$ D. Martschei, ${ }^{38}$ S. Mueller, ${ }^{38}$ Th. Müller, ${ }^{38}$ M. Niegel, ${ }^{38}$ A. Nürnberg, ${ }^{38}$ O. Oberst, ${ }^{38}$ A. Oehler, ${ }^{38}$ J. Ott, ${ }^{38}$ G. Quast, ${ }^{38}$ K. Rabbertz, ${ }^{38}$ F. Ratnikov, ${ }^{38}$ N. Ratnikova, ${ }^{38}$ S. Röcker, ${ }^{38}$ F.-P. Schilling, ${ }^{38}$ G. Schott, ${ }^{38}$ H. J. Simonis,${ }^{38}$ F. M. Stober, ${ }^{38}$ D. Troendle, ${ }^{38}$ R. Ulrich, ${ }^{38}$ J. Wagner-Kuhr, ${ }^{38}$ S. Wayand, ${ }^{38}$ T. Weiler, ${ }^{38}$ M. Zeise, ${ }^{38}$ G. Anagnostou, ${ }^{39}$ G. Daskalakis, ${ }^{39}$ T. Geralis, ${ }^{39}$ S. Kesisoglou, ${ }^{39}$ A. Kyriakis, ${ }^{39}$ D. Loukas, ${ }^{39}$ I. Manolakos, ${ }^{39}$ A. Markou, ${ }^{39}$ C. Markou, ${ }^{39}$ E. Ntomari, ${ }^{39}$ L. Gouskos ${ }^{40}$ T. J. Mertzimekis, ${ }^{40}$ A. Panagiotou,${ }^{40}$ N. Saoulidou, ${ }^{40}$ I. Evangelou, ${ }^{41}$ C. Foudas ${ }^{41}$ P. Kokkas,${ }^{41}$ N. Manthos, ${ }^{41}$ I. Papadopoulos, ${ }^{41}$ G. Bencze,${ }^{42}$ C. Hajdu, ${ }^{42}$ P. Hidas, ${ }^{42}$ D. Horvath ${ }^{42, t}$ F. Sikler, ${ }^{42}$ V. Veszpremi, ${ }^{42}$ G. Vesztergombi, ${ }^{42, \text { u }}$ A. J. Zsigmond, ${ }^{42}$ N. Beni, ${ }^{43}$ S. Czellar, ${ }^{43}$ J. Molnar, ${ }^{43}$ J. Palinkas, ${ }^{43}$ Z. Szillasi, ${ }^{43}$ J. Karancsi ${ }^{44}$ P. Raics, ${ }^{44}$ Z. L. Trocsanyi, ${ }^{44}$ B. Ujvari, ${ }^{44}$ S. B. Beri, ${ }^{45}$ V. Bhatnagar, ${ }^{45}$ N. Dhingra, ${ }^{45}$ R. Gupta, ${ }^{45}$ M. Kaur, ${ }^{45}$ M. Z. Mehta, ${ }^{45}$ M. Mittal, ${ }^{45}$ N. Nishu, ${ }^{45}$ L. K. Saini ${ }^{45}$ A. Sharma, ${ }^{45}$ J. B. Singh, ${ }^{45}$ Ashok Kumar, ${ }^{46}$ Arun Kumar ${ }^{46}$ S. Ahuja, ${ }^{46}$ A. Bhardwaj ${ }^{46}$ B. C. Choudhary, ${ }^{46}$ S. Malhotra, ${ }^{46}$ M. Naimuddin, ${ }^{46}$ K. Ranjan, ${ }^{46}$ P. Saxena, ${ }^{46}$

V. Sharma, ${ }^{46}$ R. K. Shivpuri, ${ }^{46}$ S. Banerjee, ${ }^{47}$ S. Bhattacharya, ${ }^{47}$ K. Chatterjee,${ }^{47}$ S. Dutta, ${ }^{47}$ B. Gomber, ${ }^{47}$ Sa. Jain, ${ }^{47}$ Sh. Jain, ${ }^{47}$ R. Khurana, ${ }^{47}$ A. Modak,${ }^{47}$ S. Mukherjee,${ }^{47}$ D. Roy ${ }^{47}$ S. Sarkar, ${ }^{47}$ M. Sharan, ${ }^{47}$ A. Abdulsalam, ${ }^{48}$ 
D. Dutta ${ }^{48}$ S. Kailas, ${ }^{48}$ V. Kumar, ${ }^{48}$ A. K. Mohanty, ${ }^{48, c}$ L. M. Pant, ${ }^{48}$ P. Shukla, ${ }^{48}$ T. Aziz,${ }^{49}$ R. M. Chatterjee,${ }^{49}$ S. Ganguly, ${ }^{49}$ M. Guchait, ${ }^{49, v}$ A. Gurtu ${ }^{49, w}$ M. Maity, ${ }^{49, x}$ G. Majumder, ${ }^{49}$ K. Mazumdar, ${ }^{49}$ G. B. Mohanty ${ }^{49}$ B. Parida, ${ }^{49}$ K. Sudhakar ${ }^{49}$ N. Wickramage,${ }^{49}$ S. Banerjee, ${ }^{50}$ S. Dugad ${ }^{50}$ H. Arfaei ${ }^{51, y}$ H. Bakhshiansohi, ${ }^{51}$ S. M. Etesami,${ }^{51, z}$ A. Fahim, ${ }^{51, y}$ M. Hashemi,${ }^{51, \text { aa }}$ H. Hesari, ${ }^{51}$ A. Jafari, ${ }^{51}$ M. Khakzad, ${ }^{51}$

M. Mohammadi Najafabadi, ${ }^{51}$ S. Paktinat Mehdiabadi, ${ }^{51}$ B. Safarzadeh, ${ }^{51, b b}$ M. Zeinali, ${ }^{51}$ M. Abbrescia,,${ }^{52 a, 52 b}$ L. Barbone, ${ }^{52 a, 52 b}$ C. Calabria, ${ }^{52 a, 52 b, c}$ S. S. Chhibra, ${ }^{52 a, 52 b}$ A. Colaleo, ${ }^{52 a}$ D. Creanza, ${ }^{52 a, 52 c}$ N. De Filippis, ${ }^{52 a, 52 c, c}$ M. De Palma, ${ }^{52 a, 52 b}$ L. Fiore, ${ }^{52 a}$ G. Iaselli, ${ }^{52 a, 52 c}$ G. Maggi, ${ }^{52 a, 52 c}$ M. Maggi, ${ }^{52 a}$ B. Marangelli, ${ }^{52 a, 52 b}$ S. My, ${ }^{52 a, 52 c}$ S. Nuzzo, ${ }^{52 a, 52 b}$ N. Pacifico, ${ }^{52 a}$ A. Pompili, ${ }^{52 a, 52 b}$ G. Pugliese, ${ }^{52 a, 52 c}$ G. Selvaggi, ${ }^{52 a, 52 b}$ L. Silvestris, ${ }^{52 a}$ G. Singh, ${ }^{52 a, 52 b}$ R. Venditti, ${ }^{52 a, 52 b}$ P. Verwilligen, ${ }^{52 a}$ G. Zito, ${ }^{52 a}$ G. Abbiendi, ${ }^{53 a}$ A. C. Benvenuti, ${ }^{53 a}$ D. Bonacorsi, ${ }^{53 a, 53 b}$ S. Braibant-Giacomelli, ${ }^{53 a, 53 b}$ L. Brigliadori, ${ }^{53 a, 53 b}$ P. Capiluppi, ${ }^{53 a, 53 b}$ A. Castro, ${ }^{53 a, 53 b}$ F. R. Cavallo, ${ }^{53 a}$ M. Cuffiani, ${ }^{53 a, 53 b}$ G. M. Dallavalle, ${ }^{53 a}$ F. Fabbri, ${ }^{53 a}$ A. Fanfani, ${ }^{53 a, 53 b}$ D. Fasanella, ${ }^{53 a, 53 b}$ P. Giacomelli, ${ }^{53 a}$ C. Grandi, ${ }^{53 a}$ L. Guiducci, ${ }^{53 a, 53 b}$ S. Marcellini, ${ }^{53 a}$ G. Masetti, ${ }^{53 a}$ M. Meneghelli, ${ }^{53 a, 53 b, c}$ A. Montanari, ${ }^{53 a}$ F. L. Navarria, ${ }^{53 a, 53 b}$ F. Odorici, ${ }^{53 a}$ A. Perrotta, ${ }^{53 a}$ F. Primavera, ${ }^{53 a, 53 b}$ A. M. Rossi, ${ }^{53 a, 53 b}$ T. Rovelli, ${ }^{53 a, 53 b}$ G. P. Siroli, ${ }^{53 a, 53 b}$ N. Tosi, ${ }^{53 a}$ R. Travaglini, ${ }^{53 a, 53 b}$ S. Albergo, ${ }^{54 a, 54 b}$ G. Cappello, ${ }^{54 a, 54 b}$ M. Chiorboli, ${ }^{54 a, 54 b}$ S. Costa, ${ }^{54 a, 54 b}$ R. Potenza, ${ }^{54,54 b}$ A. Tricomi, ${ }^{54 a, 54 b}$ C. Tuve, ${ }^{54 a, 54 b}$ G. Barbagli, ${ }^{55 a}$ V. Ciulli, ${ }^{55 a, 55 b}$ C. Civinini, ${ }^{55 a}$ R. D’ Alessandro, ${ }^{55 a, 55 b}$ E. Focardi, ${ }^{55 a, 55 b}$ S. Frosali, ${ }^{55 a, 55 b}$ E. Gallo, ${ }^{55 a}$ S. Gonzi, ${ }^{55 a, 55 b}$ M. Meschini, ${ }^{55 \mathrm{a}}$ S. Paoletti, ${ }^{55 \mathrm{a}}$ G. Sguazzoni, ${ }^{55 \mathrm{a}}$ A. Tropiano, ${ }^{55 \mathrm{a}, 55 \mathrm{~b}}$ L. Benussi ${ }^{56} \mathrm{~S}$. Bianco, ${ }^{56} \mathrm{~S}$. Colafranceschi, ${ }^{56, \mathrm{cc}}$ F. Fabbri, ${ }^{56}$ D. Piccolo, ${ }^{56}$ P. Fabbricatore, ${ }^{57 a}$ R. Musenich, ${ }^{57 a}$ S. Tosi,${ }^{57 a, 57 b}$ A. Benaglia, ${ }^{58 a}$ F. De Guio, ${ }^{58 a, 58 b}$ L. Di Matteo, ${ }^{58 a, 58 b, c}$ S. Fiorendi, ${ }^{58 a, 58 b}$ S. Gennai, ${ }^{58 a, c}$ A. Ghezzi, ${ }^{58 a, 58 b}$ M. T. Lucchini ${ }^{58 a, c}$ S. Malvezzi, ${ }^{58 a}$ R. A. Manzoni, ${ }^{58 a, 58 b}$ A. Martelli, ${ }^{58 a, 58 b}$ A. Massironi, ${ }^{58 a, 58 b}$ D. Menasce, ${ }^{58 a}$ L. Moroni, ${ }^{58 a}$ M. Paganoni, ${ }^{58 a, 58 b}$ D. Pedrini, ${ }^{58 a}$ S. Ragazzi, ${ }^{58 a, 58 b}$ N. Redaelli, ${ }^{58 a}$ T. Tabarelli de Fatis,${ }^{58 a, 58 b}$ S. Buontempo, ${ }^{59 a}$ N. Cavallo, ${ }^{59 a, d d}$ A. De Cosa, ${ }^{59 a, 59 b, c}$ O. Dogangun, ${ }^{59 a, 59 b}$ F. Fabozzi, ${ }^{59 a, d d}$ A. O. M. Iorio, ${ }^{59 a, 59 b}$ L. Lista, ${ }^{59 a}$ S. Meola, ${ }^{59 a, e e}$ M. Merola, ${ }^{59 a}$ P. Paolucci, ${ }^{59 a, c}$ P. Azzi, ${ }^{60 a}$ N. Bacchetta,${ }^{60 a, c}$ P. Bellan, ${ }^{60 a, 60 b}$ D. Bisello, ${ }^{60 a, 60 b}$ A. Branca, ${ }^{60 a, 60 b, c}$ R. Carlin, ${ }^{60 a, 60 b}$ P. Checchia, ${ }^{60 a}$ T. Dorigo, ${ }^{60 a}$ U. Dosselli, ${ }^{60 \mathrm{a}}$ F. Gasparini, ${ }^{60 a}, 60 \mathrm{~b}$ U. Gasparini, ${ }^{60 \mathrm{a}, 60 \mathrm{~b}}$ A. Gozzelino, ${ }^{60 \mathrm{a}}$

K. Kanishchev, ${ }^{60,60 \mathrm{c}}$ S. Lacaprara, ${ }^{60 \mathrm{a}}$ I. Lazzizzera, ${ }^{60 \mathrm{a}, 60 \mathrm{c}}$ M. Margoni, ${ }^{60 \mathrm{a}, 60 \mathrm{~b}}$ A. T. Meneguzzo, ${ }^{60 \mathrm{a}, 60 \mathrm{~b}}$

M. Nespolo, ${ }^{60 a, c}$ J. Pazzini, ${ }^{60 a, 60 b}$ P. Ronchese,${ }^{60,60 b}$ F. Simonetto, ${ }^{60 a, 60 b}$ E. Torassa, ${ }^{60 a}$ S. Vanini, ${ }^{60 a, 60 b}$ P. Zotto, ${ }^{60 a, 60 b}$ G. Zumerle, ${ }^{60 a, 60 b}$ M. Gabusi, ${ }^{61 \mathrm{a}, 61 \mathrm{~b}}$ S. P. Ratti, ${ }^{61 \mathrm{a}, 61 \mathrm{~b}}$ C. Riccardi, ${ }^{61 \mathrm{a}, 61 \mathrm{~b}}$ P. Torre, ${ }^{61 \mathrm{a}, 61 \mathrm{~b}}$ P. Vitulo, ${ }^{61 \mathrm{a}, 61 \mathrm{~b}}$ M. Biasini, ${ }^{62 a, 62 b}$ G. M. Bilei, ${ }^{62 a}$ L. Fanò,${ }^{62 a, 62 b}$ P. Lariccia, ${ }^{62 a, 62 b}$ G. Mantovani, ${ }^{62 a, 62 b}$ M. Menichelli, ${ }^{62 a}$ A. Nappi ${ }^{62 a, 62 b, a}$ F. Romeo, ${ }^{62 a, 62 b}$ A. Saha, ${ }^{62 a}$ A. Santocchia,${ }^{62 a, 62 b}$ A. Spiezia, ${ }^{62 a, 62 b}$ S. Taroni, ${ }^{62 a, 62 b}$ P. Azzurri, ${ }^{63 a, 63 c}$ G. Bagliesi, ${ }^{63 a}$ J. Bernardini, ${ }^{63 a}$ T. Boccali, ${ }^{63 a}$ G. Broccolo, ${ }^{63 a, 63 c}$ R. Castaldi, ${ }^{63 a}$ R. T. D’Agnolo, ${ }^{63 a, 63 c, c}$ R. Dell'Orso, ${ }^{63 a}$ F. Fiori, ${ }^{63 a, 63 b, c}$ L. Foà, ${ }^{63 a, 63 c}$ A. Giassi, ${ }^{63 a}$ A. Kraan, ${ }^{63 a}$ F. Ligabue, ${ }^{63 a, 63 c}$ T. Lomtadze, ${ }^{63 a}$ L. Martini, ${ }^{63 a, f f}$ A. Messineo, ${ }^{63 a, 63 b}$ F. Palla, ${ }^{63 a}$ A. Rizzi, ${ }^{63 a, 63 b}$ A. T. Serban, ${ }^{63 a, g g}$ P. Spagnolo, ${ }^{63 a}$ P. Squillacioti, ${ }^{63 a, c}$ R. Tenchini, ${ }^{63 a}$ G. Tonelli, ${ }^{63 a, 63 b}$ A. Venturi, ${ }^{63 a}$ P. G. Verdini, ${ }^{63 a}$ L. Barone,${ }^{64 a, 64 b}$ F. Cavallari, ${ }^{64 a}$ D. Del Re ${ }^{64 a, 64 b}$ M. Diemoz, ${ }^{64 a}$ C. Fanelli, ${ }^{64 a, 64 b}$ M. Grassi, ${ }^{64 a, 64 b, c}$ E. Longo, ${ }^{64 a, 64 b}$ P. Meridiani, ${ }^{64 a, c}$ F. Micheli, ${ }^{64 a, 64 b}$ S. Nourbakhsh, ${ }^{64 a, 64 b}$ G. Organtini, ${ }^{64 a, 64 b}$ R. Paramatti, ${ }^{64 a}$ S. Rahatlou, ${ }^{64 a, 64 b}$ L. Soffi, ${ }^{64 a, 64 b}$ N. Amapane ${ }^{65 a, 65 b}$ R. Arcidiacono, ${ }^{65 a, 65 \mathrm{c}}$ S. Argiro, ${ }^{65 a, 65 \mathrm{~b}} \mathrm{M}$. Arneodo, ${ }^{65 a, 65 \mathrm{c}}$ C. Biino, ${ }^{65 \mathrm{a}} \mathrm{N}$. Cartiglia, ${ }^{65 \mathrm{a}}$ S. Casasso, ${ }^{65 a, 65 b}$ M. Costa, ${ }^{65 a, 65 b}$ N. Demaria,${ }^{65 a}$ C. Mariotti, ${ }^{65 a, c}$ S. Maselli, ${ }^{65 a}$ E. Migliore ${ }^{65 a, 65 b}$ V. Monaco, ${ }^{65 a, 65 b}$ M. Musich, ${ }^{65 a, c}$ M. M. Obertino, ${ }^{65 a, 65 c}$ N. Pastrone, ${ }^{65 a}$ M. Pelliccioni, ${ }^{65 a}$ A. Potenza, ${ }^{65,65 b}$ A. Romero, ${ }^{65 a, 65 b}$ M. Ruspa,${ }^{65 a, 65 c}$ R. Sacchi, ${ }^{65 a, 65 b}$ A. Solano, ${ }^{65 a, 65 b}$ A. Staiano, ${ }^{65 a}$ S. Belforte, ${ }^{66 a}$ V. Candelise,${ }^{66 a, 66 b}$ M. Casarsa ${ }^{66 a}$

F. Cossutti, ${ }^{66, c}$ G. Della Ricca, ${ }^{66 a, 66 b}$ B. Gobbo, ${ }^{66 a}$ M. Marone, ${ }^{66 a, 66 b, c}$ D. Montanino, ${ }^{66 a, 66 b}$ A. Penzo, ${ }^{66 a}$

A. Schizzi ${ }^{66 a, 66 b}$ T. Y. Kim,${ }^{67}$ S. K. Nam, ${ }^{67}$ S. Chang, ${ }^{68}$ D. H. Kim,${ }^{68}$ G. N. Kim, ${ }^{68}$ D. J. Kong,${ }^{68}$ H. Park,${ }^{68}$ D. C. Son, ${ }^{68}$ T. Son, ${ }^{68}$ J. Y. Kim, ${ }^{69}$ Zero J. Kim, ${ }^{69}$ S. Song, ${ }^{69}$ S. Choi,${ }^{70}$ D. Gyun,${ }^{70}$ B. Hong,${ }^{70}$ M. Jo, ${ }^{70}$ H. Kim, ${ }^{70}$ T. J. Kim,${ }^{70}$ K. S. Lee, ${ }^{70}$ D. H. Moon,${ }^{70}$ S. K. Park, ${ }^{70}$ Y. Roh,${ }^{70}$ M. Choi, ${ }^{71}$ J. H. Kim, ${ }^{71}$ C. Park, ${ }^{71}$ I. C. Park,${ }^{71}$ S. Park,${ }^{71}$ G. Ryu, ${ }^{71}$ Y. Choi, ${ }^{72}$ Y. K. Choi,${ }^{72}$ J. Goh, ${ }^{72}$ M. S. Kim,${ }^{72}$ E. Kwon,${ }^{72}$ B. Lee ${ }^{72}$ J. Lee, ${ }^{72}$ S. Lee,${ }^{72}$ H. Seo, ${ }^{72}$ I. Yu, ${ }^{72}$ M. J. Bilinskas, ${ }^{73}$ I. Grigelionis, ${ }^{73}$ M. Janulis, ${ }^{73}$ A. Juodagalvis, ${ }^{73}$ H. Castilla-Valdez,${ }^{74}$ E. De La Cruz-Burelo ${ }^{74}$ I. Heredia-de La Cruz, ${ }^{74}$ R. Lopez-Fernandez, ${ }^{74}$ J. Martínez-Ortega,${ }^{74}$

A. Sánchez Hernández, ${ }^{74}$ L. M. Villasenor-Cendejas, ${ }^{74}$ S. Carrillo Moreno, ${ }^{75}$ F. Vazquez Valencia, ${ }^{75}$

H. A. Salazar Ibarguen, ${ }^{76}$ E. Casimiro Linares, ${ }^{77}$ A. Morelos Pineda, ${ }^{77}$ M. A. Reyes-Santos, ${ }^{77}$ D. Krofcheck, ${ }^{78}$ A. J. Bell, ${ }^{79}$ P. H. Butler, ${ }^{79}$ R. Doesburg, ${ }^{79}$ S. Reucroft, ${ }^{79}$ H. Silverwood, ${ }^{79}$ M. Ahmad, ${ }^{80}$ M. I. Asghar,${ }^{80}$ J. Butt, ${ }^{80}$ H. R. Hoorani, ${ }^{80}$ S. Khalid, ${ }^{80}$ W. A. Khan,${ }^{80}$ T. Khurshid, ${ }^{80}$ S. Qazi, ${ }^{80}$ M. A. Shah ${ }^{80}$ M. Shoaib,${ }^{80}$ H. Bialkowska, ${ }^{81}$ B. Boimskaż, ${ }^{81}$ T. Frueboes, ${ }^{81}$ M. Górski,${ }^{81}$ M. Kazana,${ }^{81}$ K. Nawrocki, ${ }^{81}$ K. Romanowska-Rybinska, ${ }^{81}$ 
M. Szleper ${ }^{81}$ G. Wrochna, ${ }^{81}$ P. Zalewski, ${ }^{81}$ G. Brona,${ }^{82}$ K. Bunkowski, ${ }^{82}$ M. Cwiok, ${ }^{82}$ W. Dominik, ${ }^{82}$ K. Doroba,${ }^{82}$ A. Kalinowski ${ }^{82}$ M. Konecki, ${ }^{82}$ J. Krolikowski, ${ }^{82}$ M. Misiura ${ }^{82}$ W. Wolszczak, ${ }^{82}$ N. Almeida ${ }^{83}$ P. Bargassa, ${ }^{83}$ A. David ${ }^{83}$ P. Faccioli, ${ }^{83}$ P. G. Ferreira Parracho,${ }^{83}$ M. Gallinaro, ${ }^{83}$ J. Seixas, ${ }^{83}$ J. Varela, ${ }^{83}$ P. Vischia,${ }^{83}$ I. Belotelov ${ }^{84}$ P. Bunin, ${ }^{84}$ M. Gavrilenko, ${ }^{84}$ I. Golutvin, ${ }^{84}$ I. Gorbunov,${ }^{84}$ A. Kamenev, ${ }^{84}$ V. Karjavin, ${ }^{84}$ G. Kozlov, ${ }^{84}$ A. Lanev, ${ }^{84}$ A. Malakhov, ${ }^{84}$ P. Moisenz, ${ }^{84}$ V. Palichik, ${ }^{84}$ V. Perelygin, ${ }^{84}$ S. Shmatov,${ }^{84}$ V. Smirnov, ${ }^{84}$ A. Volodko, ${ }^{84}$ A. Zarubin, ${ }^{84}$ S. Evstyukhin, ${ }^{85}$ V. Golovtsov, ${ }^{85}$ Y. Ivanov, ${ }^{85}$ V. Kim, ${ }^{85}$ P. Levchenko, ${ }^{85}$ V. Murzin, ${ }^{85}$ V. Oreshkin, ${ }^{85}$ I. Smirnov, ${ }^{85}$ V. Sulimov, ${ }^{85}$ L. Uvarov, ${ }^{85}$ S. Vavilov, ${ }^{85}$ A. Vorobyev,${ }^{85}$ An. Vorobyev, ${ }^{85}$ Yu. Andreev, ${ }^{86}$

A. Dermenev ${ }^{86}$ S. Gninenko, ${ }^{86}$ N. Golubev, ${ }^{86}$ M. Kirsanov, ${ }^{86}$ N. Krasnikov, ${ }^{86}$ V. Matveev, ${ }^{86}$ A. Pashenkov, ${ }^{86}$ D. Tlisov, ${ }^{86}$ A. Toropin, ${ }^{86}$ V. Epshteyn,${ }^{87}$ M. Erofeeva, ${ }^{87}$ V. Gavrilov, ${ }^{87}$ M. Kossov,${ }^{87}$ N. Lychkovskaya, ${ }^{87}$ V. Popov, ${ }^{87}$ G. Safronov, ${ }^{87}$ S. Semenov, ${ }^{87}$ I. Shreyber, ${ }^{87}$ V. Stolin, ${ }^{87}$ E. Vlasov, ${ }^{87}$ A. Zhokin, ${ }^{87}$ A. Belyaev, ${ }^{88}$ E. Boos, ${ }^{88}$ M. Dubinin, ${ }^{88, f}$ L. Dudko, ${ }^{88}$ A. Ershov, ${ }^{88}$ A. Gribushin,${ }^{88}$ V. Klyukhin, ${ }^{88}$ O. Kodolova, ${ }^{88}$ I. Lokhtin, ${ }^{88}$ A. Markina ${ }^{88}$ S. Obraztsov, ${ }^{88}$ M. Perfilov ${ }^{88}$ S. Petrushanko, ${ }^{88}$ A. Popov ${ }^{88}$ L. Sarycheva,${ }^{88, a}$ V. Savrin, ${ }^{88}$ A. Snigirev,${ }^{88}$ V. Andreev ${ }^{89}$ M. Azarkin, ${ }^{89}$ I. Dremin, ${ }^{89}$ M. Kirakosyan, ${ }^{89}$ A. Leonidov, ${ }^{89}$ G. Mesyats, ${ }^{89}$ S. V. Rusakov, ${ }^{89}$ A. Vinogradov,${ }^{89}$ I. Azhgirey, ${ }^{90}$ I. Bayshev, ${ }^{90}$ S. Bitioukov,${ }^{90}$ V. Grishin, ${ }^{90, \mathrm{c}}$ V. Kachanov, ${ }^{90}$ D. Konstantinov, ${ }^{90}$ V. Krychkine, ${ }^{90}$ V. Petrov, ${ }^{90}$ R. Ryutin, ${ }^{90}$ A. Sobol, ${ }^{90}$ L. Tourtchanovitch, ${ }^{90}$ S. Troshin, ${ }^{90}$ N. Tyurin, ${ }^{90}$ A. Uzunian, ${ }^{90}$ A. Volkov, ${ }^{90}$ P. Adzic, ${ }^{91, \text { hh }}$ M. Djordjevic, ${ }^{91}$ M. Ekmedzic, ${ }^{91}$ D. Krpic,${ }^{91, \text { hh }}$ J. Milosevic, ${ }^{91}$ M. Aguilar-Benitez, ${ }^{92}$ J. Alcaraz Maestre, ${ }^{92}$ P. Arce, ${ }^{92}$ C. Battilana, ${ }^{92}$ E. Calvo, ${ }^{92}$ M. Cerrada, ${ }^{92}$ M. Chamizo Llatas, ${ }^{92}$ N. Colino, ${ }^{92}$ B. De La Cruz,${ }^{92}$ A. Delgado Peris, ${ }^{92}$ D. Domínguez Vázquez,${ }^{92}$ C. Fernandez Bedoya,${ }^{92}$ J. P. Fernández Ramos, ${ }^{92}$ A. Ferrando,${ }^{92}$ J. Flix,${ }^{92}$ M. C. Fouz,${ }^{92}$ P. Garcia-Abia, ${ }^{92}$ O. Gonzalez Lopez, ${ }^{92}$ S. Goy Lopez, ${ }^{92}$ J. M. Hernandez,${ }^{92}$ M. I. Josa ${ }^{92}$ G. Merino, ${ }^{92}$ J. Puerta Pelayo, ${ }^{92}$ A. Quintario Olmeda,${ }^{92}$ I. Redondo, ${ }^{92}$ L. Romero, ${ }^{92}$ J. Santaolalla, ${ }^{92}$ M. S. Soares,${ }^{92}$ C. Willmott, ${ }^{92}$ C. Albajar, ${ }^{93}$ G. Codispoti, ${ }^{93}$ J. F. de Trocóniz, ${ }^{93}$ H. Brun, ${ }^{94}$ J. Cuevas, ${ }^{94}$

J. Fernandez Menendez, ${ }^{94}$ S. Folgueras,${ }^{94}$ I. Gonzalez Caballero, ${ }^{94}$ L. Lloret Iglesias, ${ }^{94}$ J. Piedra Gomez, ${ }^{94}$ J. A. Brochero Cifuentes, ${ }^{95}$ I. J. Cabrillo, ${ }^{95}$ A. Calderon, ${ }^{95}$ S. H. Chuang, ${ }^{95}$ J. Duarte Campderros, ${ }^{95}$ M. Felcini, ${ }^{95, \text { ii }}$ M. Fernandez, ${ }^{95}$ G. Gomez,${ }^{95}$ J. Gonzalez Sanchez,${ }^{95}$ A. Graziano,${ }^{95}$ C. Jorda, ${ }^{95}$ A. Lopez Virto, ${ }^{95}$ J. Marco, ${ }^{95}$ R. Marco, ${ }^{95}$ C. Martinez Rivero, ${ }^{95}$ F. Matorras, ${ }^{95}$ F. J. Munoz Sanchez, ${ }^{95}$ T. Rodrigo, ${ }^{95}$ A. Y. Rodríguez-Marrero, ${ }^{95}$ A. Ruiz-Jimeno, ${ }^{95}$ L. Scodellaro, ${ }^{95}$ I. Vila,${ }^{95}$ R. Vilar Cortabitarte, ${ }^{95}$ D. Abbaneo, ${ }^{96}$ E. Auffray, ${ }^{96}$ G. Auzinger,${ }^{96}$ M. Bachtis, ${ }^{96}$ P. Baillon, ${ }^{96}$ A. H. Ball,${ }^{96}$ D. Barney, ${ }^{96}$ J. F. Benitez, ${ }^{96}$ C. Bernet,${ }^{96,9}$ G. Bianchi, ${ }^{96}$ P. Bloch, ${ }^{96}$ A. Bocci, ${ }^{96}$ A. Bonato, ${ }^{96}$ C. Botta,${ }^{96}$ H. Breuker, ${ }^{96}$ T. Camporesi,${ }^{96}$ G. Cerminara,,${ }^{96}$ T. Christiansen, ${ }^{96}$ J. A. Coarasa Perez,${ }^{96}$ D. D’Enterria, ${ }^{96}$ A. Dabrowski, ${ }^{96}$ A. De Roeck, ${ }^{96}$ S. Di Guida, ${ }^{96}$ M. Dobson,,${ }^{96}$ N. Dupont-Sagorin, ${ }^{96}$ A. Elliott-Peisert, ${ }^{96}$ B. Frisch, ${ }^{96}$ W. Funk,${ }^{96}$ G. Georgiou, ${ }^{96}$ M. Giffels,${ }^{96}$ D. Gigi,${ }^{96}$ K. Gill, ${ }^{96}$ D. Giordano,${ }^{96} \mathrm{M}$. Girone,${ }^{96} \mathrm{M}$. Giunta,${ }^{96}$ F. Glege,${ }^{96} \mathrm{R}$. Gomez-Reino Garrido, ${ }^{96}$ P. Govoni, ${ }^{96} \mathrm{~S}$. Gowdy, ${ }^{96}$ R. Guida, ${ }^{96}$ J. Hammer, ${ }^{96}$ M. Hansen, ${ }^{96}$ P. Harris,${ }^{96}$ C. Hartl, ${ }^{96}$ J. Harvey,${ }^{96}$ B. Hegner, ${ }^{96}$ A. Hinzmann, ${ }^{96}$ V. Innocente, ${ }^{96}$ P. Janot,${ }^{96}$ K. Kaadze,${ }^{96}$ E. Karavakis, ${ }^{96}$ K. Kousouris, ${ }^{96}$ P. Lecoq, ${ }^{96}$ Y.-J. Lee, ${ }^{96}$ P. Lenzi,,${ }^{96}$ C. Lourenço, ${ }^{96}$ N. Magini, ${ }^{96}$ T. Mäki,${ }^{96}$ M. Malberti, ${ }^{96}$ L. Malgeri,${ }^{96}$ M. Mannelli, ${ }^{96}$ L. Masetti, ${ }^{96}$ F. Meijers, ${ }^{96}$ S. Mersi, ${ }^{96}$ E. Meschi, ${ }^{96}$ R. Moser,${ }^{96}$ M. Mulders, ${ }^{96}$ P. Musella, ${ }^{96}$ E. Nesvold ${ }^{96}$ L. Orsini, ${ }^{96}$ E. Palencia Cortezon, ${ }^{96}$ E. Perez,${ }^{96}$ L. Perrozzi, ${ }^{96}$ A. Petrilli, ${ }^{96}$ A. Pfeiffer,${ }^{96}$ M. Pierini, ${ }^{96}$ M. Pimiä, ${ }^{96}$ D. Piparo, ${ }^{96}$ G. Polese,${ }^{96}$ L. Quertenmont, ${ }^{96}$ A. Racz, ${ }^{96}$ W. Reece, ${ }^{96}$ J. Rodrigues Antunes, ${ }^{96}$ G. Rolandi, ${ }^{96, j j}$ C. Rovelli, ${ }^{96, k k}$ M. Rovere, ${ }^{96}$ H. Sakulin, ${ }^{96}$ F. Santanastasio, ${ }^{96}$ C. Schäfer, ${ }^{96}$ C. Schwick, ${ }^{96}$ I. Segoni, ${ }^{96}$ S. Sekmen, ${ }^{96}$ A. Sharma, ${ }^{96}$ P. Siegrist, ${ }^{96}$ P. Silva, ${ }^{96}$ M. Simon, ${ }^{96}$ P. Sphicas, ${ }^{96,11}$ D. Spiga, ${ }^{96}$ A. Tsirou, ${ }^{96}$ G. I. Veres,${ }^{96, u}$ J. R. Vlimant ${ }^{96}$ H. K. Wöhri, ${ }^{96}$ S. D. Worm, ${ }^{96, \mathrm{~mm}}$ W. D. Zeuner, ${ }^{96}$ W. Bertl, ${ }^{97}$ K. Deiters, ${ }^{97}$ W. Erdmann, ${ }^{97}$ K. Gabathuler, ${ }^{97}$ R. Horisberger, ${ }^{97}$ Q. Ingram, ${ }^{97}$ H. C. Kaestli,${ }^{97}$ S. König,${ }^{97}$ D. Kotlinski,,${ }^{97}$ U. Langenegger, ${ }^{97}$ F. Meier, ${ }^{97}$ D. Renker, ${ }^{97}$ T. Rohe, ${ }^{97}$ F. Bachmair ${ }^{98}$ L. Bäni,${ }^{98}$ P. Bortignon, ${ }^{98}$ M. A. Buchmann, ${ }^{98}$ B. Casal,${ }^{98}$ N. Chanon, ${ }^{98}$ A. Deisher, ${ }^{98}$ G. Dissertori,${ }^{98}$ M. Dittmar, ${ }^{98}$ M. Donegà, ${ }^{98}$ M. Dünser, ${ }^{98}$ P. Eller,${ }^{98}$ J. Eugster,${ }^{98}$ K. Freudenreich,,${ }^{98}$ C. Grab, ${ }^{98}$ D. Hits,,${ }^{98}$ P. Lecomte, ${ }^{98}$ W. Lustermann, ${ }^{98}$ A. C. Marini, ${ }^{98}$ P. Martinez Ruiz del Arbol, ${ }^{98}$ N. Mohr,${ }^{98}$ F. Moortgat, ${ }^{98}$ C. Nägeli, ${ }^{98, n n}$ P. Nef,${ }^{98}$ F. Nessi-Tedaldi,${ }^{98}$ F. Pandolfi, ${ }^{98}$ L. Pape,${ }^{98}$ F. Pauss, ${ }^{98}$ M. Peruzzi, ${ }^{98}$ F. J. Ronga,${ }^{98}$ M. Rossini,${ }^{98}$ L. Sala, ${ }^{98}$ A. K. Sanchez,${ }^{98}$ A. Starodumov, ${ }^{98,00}$ B. Stieger, ${ }^{98}$ M. Takahashi, ${ }^{98}$ L. Tauscher,,${ }^{98, a}$ A. Thea, ${ }^{98}$ K. Theofilatos, ${ }^{98}$ D. Treille, ${ }^{98}$ C. Urscheler, ${ }^{98}$ R. Wallny, ${ }^{98}$ H. A. Weber,${ }^{98}$ L. Wehrli, ${ }^{98}$ C. Amsler, ${ }^{99, p p}$ V. Chiochia, ${ }^{99}$ S. De Visscher, ${ }^{99}$ C. Favaro,${ }^{99}$ M. Ivova Rikova, ${ }^{99}$ B. Kilminster, ${ }^{99}$ B. Millan Mejias, ${ }^{99}$ P. Otiougova, ${ }^{99}$ P. Robmann, ${ }^{99}$ H. Snoek, ${ }^{99}$ S. Tupputi, ${ }^{99}$ M. Verzetti, ${ }^{99}$ Y. H. Chang, ${ }^{100}$ K. H. Chen, ${ }^{100}$ C. Ferro, ${ }^{100}$ C. M. Kuo, ${ }^{100}$ S. W. Li,${ }^{100}$ W. Lin,${ }^{100}$ Y. J. Lu, ${ }^{100}$ A. P. Singh, ${ }^{100}$ R. Volpe, ${ }^{100}$ S. S. Yu, ${ }^{100}$ P. Bartalini, ${ }^{101}$ P. Chang,,${ }^{101}$ Y. H. Chang, ${ }^{101}$ Y. W. Chang,,${ }^{101}$ Y. Chao, ${ }^{101}$ K. F. Chen, ${ }^{101}$ C. Dietz, ${ }^{101}$ U. Grundler,${ }^{101}$ W.-S. Hou, ${ }^{101}$ Y. Hsiung, ${ }^{101}$ 
K. Y. Kao,${ }^{101}$ Y. J. Lei, ${ }^{101}$ R.-S. Lu, ${ }^{101}$ D. Majumder, ${ }^{101}$ E. Petrakou, ${ }^{101}$ X. Shi, ${ }^{101}$ J. G. Shiu, ${ }^{101}$ Y. M. Tzeng,${ }^{101}$ X. Wan, ${ }^{101}$ M. Wang,,${ }^{101}$ B. Asavapibhop, ${ }^{102}$ E. Simili, ${ }^{102}$ N. Srimanobhas, ${ }^{102}$ N. Suwonjandee, ${ }^{102}$ A. Adiguzel, ${ }^{103}$ M. N. Bakirci, ${ }^{103, q q}$ S. Cerci, ${ }^{103, \text { rr }}$ C. Dozen, ${ }^{103}$ I. Dumanoglu, ${ }^{103}$ E. Eskut, ${ }^{103}$ S. Girgis, ${ }^{103}$ G. Gokbulut, ${ }^{103}$ E. Gurpinar, ${ }^{103}$ I. Hos, ${ }^{103}$ E. E. Kangal, ${ }^{103}$ T. Karaman, ${ }^{103}$ G. Karapinar, ${ }^{103, s s}$ A. Kayis Topaksu, ${ }^{103}$ G. Onengut, ${ }^{103}$

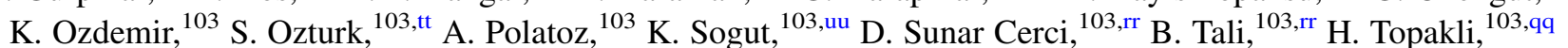
L. N. Vergili, ${ }^{103}$ M. Vergili, ${ }^{103}$ I. V. Akin, ${ }^{104}$ T. Aliev, ${ }^{104}$ B. Bilin, ${ }^{104}$ S. Bilmis,${ }^{104}$ M. Deniz ${ }^{104}$ H. Gamsizkan, ${ }^{104}$ A. M. Guler, ${ }^{104}$ K. Ocalan, ${ }^{104}$ A. Ozpineci, ${ }^{104}$ M. Serin, ${ }^{104}$ R. Sever, ${ }^{104}$ U. E. Surat, ${ }^{104}$ M. Yalvac, ${ }^{104}$ E. Yildirim, ${ }^{104}$ M. Zeyrek, ${ }^{104}$ E. Gülmez, ${ }^{105}$ B. Isildak, ${ }^{105, v v}$ M. Kaya, ${ }^{105, w w}$ O. Kaya, ${ }^{105, w w}$ S. Ozkorucuklu, ${ }^{105, \text {,x }}$ N. Sonmez, ${ }^{105, y y}$ H. Bahtiyar, ${ }^{106, z z}$ E. Barlas, ${ }^{106}$ K. Cankocak, ${ }^{106}$ Y. O. Günaydin, ${ }^{106, \text { aaa }}$ F. I. Vardarlı, ${ }^{106}$ M. Yücel, ${ }^{106}$ L. Levchuk, ${ }^{107}$ J. J. Brooke, ${ }^{108}$ E. Clement, ${ }^{108}$ D. Cussans, ${ }^{108}$ H. Flacher, ${ }^{108}$ R. Frazier, ${ }^{108}$ J. Goldstein, ${ }^{108}$ M. Grimes,${ }^{108}$ G. P. Heath, ${ }^{108}$ H. F. Heath, ${ }^{108}$ L. Kreczko, ${ }^{108}$ S. Metson, ${ }^{108}$ D. M. Newbold, ${ }^{108, m m}$ K. Nirunpong, ${ }^{108}$ A. Poll, ${ }^{108}$ S. Senkin, ${ }^{108}$ V. J. Smith, ${ }^{108}$ T. Williams, ${ }^{108}$ L. Basso, ${ }^{109, b b b}$ K. W. Bell, ${ }^{109}$ A. Belyaev, ${ }^{109, b b b}$ C. Brew, ${ }^{109}$ R. M. Brown, ${ }^{109}$ D. J. A. Cockerill, ${ }^{109}$ J. A. Coughlan, ${ }^{109}$ K. Harder, ${ }^{109}$ S. Harper, ${ }^{109}$ J. Jackson, ${ }^{109}$ B. W. Kennedy, ${ }^{109}$ E. Olaiya, ${ }^{109}$ D. Petyt,${ }^{109}$ B. C. Radburn-Smith, ${ }^{109}$ C. H. Shepherd-Themistocleous, ${ }^{109}$ I. R. Tomalin, ${ }^{109}$ W. J. Womersley, ${ }^{109}$ R. Bainbridge, ${ }^{110}$ G. Ball,${ }^{110}$ R. Beuselinck, ${ }^{110}$ O. Buchmuller, ${ }^{110}$ D. Colling, ${ }^{110}$ N. Cripps,${ }^{110}$ M. Cutajar, ${ }^{110}$ P. Dauncey, ${ }^{110}$ G. Davies,${ }^{110}$ M. Della Negra ${ }^{110}$ W. Ferguson, ${ }^{110}$ J. Fulcher, ${ }^{110}$ D. Futyan, ${ }^{110}$ A. Gilbert ${ }^{110}$ A. Guneratne Bryer, ${ }^{110}$ G. Hall ${ }^{110}$ Z. Hatherell, ${ }^{110}$ J. Hays,${ }^{110}$ G. Iles, ${ }^{110}$ M. Jarvis, ${ }^{110}$ G. Karapostoli, ${ }^{110}$ M. Kenzie, ${ }^{110}$ L. Lyons, ${ }^{110}$ A.-M. Magnan, ${ }^{110}$ J. Marrouche, ${ }^{110}$ B. Mathias, ${ }^{10}$ R. Nandi, ${ }^{110}$ J. Nash, ${ }^{110}$ A. Nikitenko, ${ }^{10,00}$ J. Pela, ${ }^{110}$ M. Pesaresi, ${ }^{110}$ K. Petridis, ${ }^{110}$ M. Pioppi, ${ }^{11,, c c c}$ D. M. Raymond, ${ }^{110}$ S. Rogerson, ${ }^{110}$ A. Rose,${ }^{110}$ C. Seez,${ }^{110}$ P. Sharp,${ }^{110, a}$ A. Sparrow, ${ }^{110}$ M. Stoye, ${ }^{110}$ A. Tapper, ${ }^{110}$ M. Vazquez Acosta, ${ }^{110}$ T. Virdee, ${ }^{110}$ S. Wakefield, ${ }^{110}$ N. Wardle, ${ }^{110}$ T. Whyntie,${ }^{110}$ M. Chadwick,${ }^{111}$ J. E. Cole, ${ }^{111}$ P. R. Hobson, ${ }^{111}$ A. Khan, ${ }^{111}$ P. Kyberd, ${ }^{111}$ D. Leggat,${ }^{111}$ D. Leslie, ${ }^{111}$ W. Martin, ${ }^{111}$ I. D. Reid, ${ }^{111}$ P. Symonds,${ }^{111}$ L. Teodorescu, ${ }^{111}$ M. Turner, ${ }^{11}$ K. Hatakeyama, ${ }^{112}$ H. Liu, ${ }^{112}$ T. Scarborough, ${ }^{112}$ O. Charaf, ${ }^{113}$ S. I. Cooper,${ }^{113}$ C. Henderson, ${ }^{113}$ P. Rumerio, ${ }^{113}$ A. Avetisyan, ${ }^{114}$ T. Bose, ${ }^{114}$ C. Fantasia, ${ }^{114}$ A. Heister, ${ }^{114}$ J. St. John, ${ }^{114}$ P. Lawson, ${ }^{114}$ D. Lazic, ${ }^{114}$ J. Rohlf, ${ }^{114}$ D. Sperka, ${ }^{114}$ L. Sulak, ${ }^{114}$ J. Alimena, ${ }^{115}$ S. Bhattacharya, ${ }^{115}$ G. Christopher, ${ }^{115}$ D. Cutts, ${ }^{115}$ Z. Demiragli, ${ }^{115}$ A. Ferapontov, ${ }^{115}$ A. Garabedian, ${ }^{115}$ U. Heintz, ${ }^{115}$ S. Jabeen, ${ }^{115}$ G. Kukartsev, ${ }^{115}$ E. Laird, ${ }^{115}$ G. Landsberg, ${ }^{115}$ M. Luk, ${ }^{115}$ M. Narain, ${ }^{115}$ M. Segala, ${ }^{115}$ T. Sinthuprasith,,${ }^{115}$ T. Speer, ${ }^{115}$ R. Breedon, ${ }^{116}$ G. Breto, ${ }^{116}$ M. Calderon De La Barca Sanchez, ${ }^{116}$ S. Chauhan, ${ }^{116}$ M. Chertok, ${ }^{116}$ J. Conway, ${ }^{116}$ R. Conway, ${ }^{116}$ P. T. Cox, ${ }^{116}$ J. Dolen, ${ }^{116}$ R. Erbacher, ${ }^{116}$ M. Gardner, ${ }^{116}$ R. Houtz, ${ }^{116}$ W. Ko, ${ }^{116}$ A. Kopecky, ${ }^{116}$ R. Lander, ${ }^{116}$ O. Mall, ${ }^{116}$ T. Miceli, ${ }^{116}$ R. Nelson, ${ }^{116}$ D. Pellett, ${ }^{116}$ F. Ricci-Tam, ${ }^{116}$ B. Rutherford,${ }^{116}$ M. Searle, ${ }^{116}$ J. Smith,${ }^{116}$ M. Squires, ${ }^{116}$ M. Tripathi, ${ }^{116}$ R. Vasquez Sierra, ${ }^{116}$ R. Yohay, ${ }^{116}$ V. Andreev,,${ }^{117}$ D. Cline,${ }^{117}$ R. Cousins, ${ }^{117}$ J. Duris, ${ }^{117}$ S. Erhan,,${ }^{117}$ P. Everaerts, ${ }^{117}$ C. Farrell, ${ }^{117}$ J. Hauser, ${ }^{117}$ M. Ignatenko, ${ }^{117}$ C. Jarvis, ${ }^{117}$ G. Rakness, ${ }^{117}$ P. Schlein, ${ }^{117, a}$ P. Traczyk, ${ }^{117}$ V. Valuev, ${ }^{117}$ M. Weber, ${ }^{117}$ J. Babb,${ }^{18}$ R. Clare, ${ }^{118}$ M. E. Dinardo, ${ }^{118}$ J. Ellison, ${ }^{118}$ J. W. Gary, ${ }^{118}$ F. Giordano, ${ }^{118}$ G. Hanson, ${ }^{118}$ H. Liu, ${ }^{118}$ O. R. Long, ${ }^{118}$ A. Luthra, ${ }^{118}$ H. Nguyen, ${ }^{118}$ S. Paramesvaran, ${ }^{118}$ J. Sturdy,${ }^{118}$ S. Sumowidagdo, ${ }^{118}$ R. Wilken, ${ }^{118}$ S. Wimpenny, ${ }^{118}$ W. Andrews,${ }^{119}$ J. G. Branson, ${ }^{119}$ G. B. Cerati, ${ }^{119}$ S. Cittolin, ${ }^{119}$ D. Evans, ${ }^{119}$ A. Holzner, ${ }^{119}$ R. Kelley, ${ }^{119}$ M. Lebourgeois, ${ }^{119}$ J. Letts, ${ }^{119}$ I. Macneill, ${ }^{119}$ B. Mangano, ${ }^{119}$ S. Padhi, ${ }^{119}$ C. Palmer,${ }^{19}$ G. Petrucciani, ${ }^{119}$ M. Pieri, ${ }^{119}$ M. Sani, ${ }^{119}$ V. Sharma, ${ }^{119}$ S. Simon, ${ }^{119}$ E. Sudano, ${ }^{119}$ M. Tadel, ${ }^{119}$ Y. Tu, ${ }^{119}$ A. Vartak, ${ }^{119}$ S. Wasserbaech,,${ }^{119, \text { ddd }}$

F. Würthwein, ${ }^{119}$ A. Yagil, ${ }^{119}$ J. Yoo, ${ }^{119}$ D. Barge, ${ }^{120}$ R. Bellan, ${ }^{120}$ C. Campagnari, ${ }^{120}$ M. D’ Alfonso, ${ }^{120}$ T. Danielson, ${ }^{120}$ K. Flowers, ${ }^{120}$ P. Geffert, ${ }^{120}$ C. George, ${ }^{120}$ F. Golf, ${ }^{120}$ J. Incandela, ${ }^{120}$ C. Justus, ${ }^{120}$ P. Kalavase, ${ }^{120}$ D. Kovalskyi, ${ }^{120}$ V. Krutelyov, ${ }^{120}$ S. Lowette, ${ }^{120}$ R. Magaña Villalba, ${ }^{120}$ N. Mccoll, ${ }^{120}$ V. Pavlunin, ${ }^{120}$ J. Ribnik, ${ }^{120}$ J. Richman, ${ }^{120}$ R. Rossin, ${ }^{120}$ D. Stuart, ${ }^{120}$ W. To, ${ }^{120}$ C. West,${ }^{120}$ A. Apresyan, ${ }^{121}$ A. Bornheim, ${ }^{121}$ Y. Chen, ${ }^{121}$ E. Di Marco, ${ }^{121}$ J. Duarte, ${ }^{121}$ M. Gataullin, ${ }^{121}$ Y. Ma,${ }^{121}$ A. Mott, ${ }^{121}$ H. B. Newman, ${ }^{121}$ C. Rogan, ${ }^{121}$ M. Spiropulu, ${ }^{121}$ V. Timciuc, ${ }^{121}$ J. Veverka, ${ }^{121}$ R. Wilkinson, ${ }^{121}$ S. Xie, ${ }^{121}$ Y. Yang, ${ }^{121}$ R. Y. Zhu, ${ }^{121}$ V. Azzolini, ${ }^{122}$ A. Calamba, ${ }^{122}$ R. Carroll, ${ }^{122}$ T. Ferguson, ${ }^{122}$ Y. Iiyama, ${ }^{122}$ D. W. Jang, ${ }^{122}$ Y. F. Liu, ${ }^{122}$ M. Paulini, ${ }^{122}$ H. Vogel, ${ }^{122}$ I. Vorobiev, ${ }^{122}$ J. P. Cumalat, ${ }^{123}$ B. R. Drell, ${ }^{123}$ W. T. Ford, ${ }^{123}$ A. Gaz, ${ }^{123}$ E. Luiggi Lopez, ${ }^{123}$ J. G. Smith, ${ }^{123}$ K. Stenson, ${ }^{123}$ K. A. Ulmer, ${ }^{123}$ S. R. Wagner, ${ }^{123}$ J. Alexander, ${ }^{124}$ A. Chatterjee, ${ }^{124}$ N. Eggert, ${ }^{124}$ L. K. Gibbons, ${ }^{124}$ B. Heltsley, ${ }^{124}$ W. Hopkins, ${ }^{124}$ A. Khukhunaishvili, ${ }^{124}$ B. Kreis, ${ }^{124}$ N. Mirman, ${ }^{124}$ G. Nicolas Kaufman, ${ }^{124}$ J. R. Patterson, ${ }^{124}$ A. Ryd, ${ }^{124}$ E. Salvati, ${ }^{124}$ W. Sun, ${ }^{124}$ W. D. Teo, ${ }^{124}$ J. Thom, ${ }^{124}$ J. Thompson, ${ }^{124}$ J. Tucker, ${ }^{124}$ J. Vaughan, ${ }^{124}$ Y. Weng, ${ }^{124}$ L. Winstrom, ${ }^{124}$ P. Wittich, ${ }^{124}$ D. Winn, ${ }^{125}$ S. Abdullin, ${ }^{126}$ M. Albrow, ${ }^{126}$ J. Anderson, ${ }^{126}$ L. A. T. Bauerdick, ${ }^{126}$ A. Beretvas, ${ }^{126}$ J. Berryhill, ${ }^{126}$ P. C. Bhat, ${ }^{126}$ K. Burkett, ${ }^{126}$ J. N. Butler, ${ }^{126}$ V. Chetluru, ${ }^{126}$ 
H. W. K. Cheung, ${ }^{126}$ F. Chlebana, ${ }^{126}$ V. D. Elvira, ${ }^{126}$ I. Fisk, ${ }^{126}$ J. Freeman, ${ }^{126}$ Y. Gao, ${ }^{126}$ D. Green, ${ }^{126}$ O. Gutsche, ${ }^{126}$ J. Hanlon, ${ }^{126}$ R. M. Harris, ${ }^{126}$ J. Hirschauer, ${ }^{126}$ B. Hooberman, ${ }^{126}$ S. Jindariani, ${ }^{126}$ M. Johnson, ${ }^{126}$ U. Joshi, ${ }^{126}$

B. Klima, ${ }^{126}$ S. Kunori, ${ }^{126}$ S. Kwan, ${ }^{126}$ C. Leonidopoulos, ${ }^{126, \text { eee }}$ J. Linacre, ${ }^{126}$ D. Lincoln, ${ }^{126}$ R. Lipton, ${ }^{126}$ J. Lykken, ${ }^{126}$ K. Maeshima, ${ }^{126}$ J. M. Marraffino, ${ }^{126}$ V. I. Martinez Outschoorn, ${ }^{126}$ S. Maruyama, ${ }^{126}$ D. Mason, ${ }^{126}$

P. McBride, ${ }^{126}$ K. Mishra, ${ }^{126}$ S. Mrenna, ${ }^{126}$ Y. Musienko, ${ }^{126, f f f}$ C. Newman-Holmes, ${ }^{126}$ V. O’Dell, ${ }^{126}$

O. Prokofyev, ${ }^{126}$ E. Sexton-Kennedy, ${ }^{126}$ S. Sharma, ${ }^{126}$ W. J. Spalding, ${ }^{126}$ L. Spiegel, ${ }^{126}$ L. Taylor, ${ }^{126}$ S. Tkaczyk, ${ }^{126}$ N. V. Tran, ${ }^{126}$ L. Uplegger, ${ }^{126}$ E. W. Vaandering, ${ }^{126}$ R. Vidal, ${ }^{126}$ J. Whitmore, ${ }^{126}$ W. Wu, ${ }^{126}$ F. Yang, ${ }^{126}$ J. C. Yun, ${ }^{126}$ D. Acosta, ${ }^{127}$ P. Avery, ${ }^{127}$ D. Bourilkov, ${ }^{127}$ M. Chen, ${ }^{127}$ T. Cheng, ${ }^{127}$ S. Das, ${ }^{127}$ M. De Gruttola, ${ }^{127}$

G. P. Di Giovanni, ${ }^{127}$ D. Dobur, ${ }^{127}$ A. Drozdetskiy, ${ }^{127}$ R. D. Field,${ }^{127}$ M. Fisher, ${ }^{127}$ Y. Fu, ${ }^{127}$ I. K. Furic,${ }^{127}$

J. Gartner, ${ }^{127}$ J. Hugon, ${ }^{127}$ B. Kim, ${ }^{127}$ J. Konigsberg, ${ }^{127}$ A. Korytov, ${ }^{127}$ A. Kropivnitskaya, ${ }^{127}$ T. Kypreos, ${ }^{127}$ J. F. Low, ${ }^{127}$ K. Matchev, ${ }^{127}$ P. Milenovic, ${ }^{127, \text { ggg }}$ G. Mitselmakher, ${ }^{127}$ L. Muniz, ${ }^{127}$ M. Park, ${ }^{127}$ R. Remington, ${ }^{127}$ A. Rinkevicius, ${ }^{127}$ P. Sellers, ${ }^{127}$ N. Skhirtladze,${ }^{127}$ M. Snowball, ${ }^{127}$ J. Yelton, ${ }^{127}$ M. Zakaria, ${ }^{127}$ V. Gaultney, ${ }^{128}$ S. Hewamanage, ${ }^{128}$ L. M. Lebolo, ${ }^{128}$ S. Linn, ${ }^{128}$ P. Markowitz,${ }^{128}$ G. Martinez, ${ }^{128}$ J. L. Rodriguez, ${ }^{128}$ T. Adams, ${ }^{129}$ A. Askew, ${ }^{129}$ J. Bochenek, ${ }^{129}$ J. Chen, ${ }^{129}$ B. Diamond, ${ }^{129}$ S. V. Gleyzer, ${ }^{129}$ J. Haas, ${ }^{129}$ S. Hagopian, ${ }^{129}$ V. Hagopian, ${ }^{129}$ M. Jenkins, ${ }^{129}$ K. F. Johnson, ${ }^{129}$ H. Prosper, ${ }^{129}$ V. Veeraraghavan, ${ }^{129}$ M. Weinberg, ${ }^{129}$ M. M. Baarmand, ${ }^{130}$ B. Dorney, ${ }^{130}$ M. Hohlmann, ${ }^{130}$ H. Kalakhety, ${ }^{130}$ I. Vodopiyanov, ${ }^{130}$ F. Yumiceva, ${ }^{130}$ M. R. Adams, ${ }^{131}$ L. Apanasevich, ${ }^{131}$ Y. Bai, ${ }^{131}$ V.E. Bazterra, ${ }^{131}$ R. R. Betts, ${ }^{131}$ I. Bucinskaite, ${ }^{131}$ J. Callner, ${ }^{131}$ R. Cavanaugh, ${ }^{131}$ O. Evdokimov, ${ }^{131}$ L. Gauthier, ${ }^{131}$ C. E. Gerber, ${ }^{131}$ D. J. Hofman, ${ }^{131}$ S. Khalatyan, ${ }^{131}$ F. Lacroix, ${ }^{131}$

C. O'Brien, ${ }^{131}$ C. Silkworth, ${ }^{131}$ D. Strom, ${ }^{131}$ P. Turner, ${ }^{131}$ N. Varelas, ${ }^{131}$ U. Akgun, ${ }^{132}$ E. A. Albayrak,${ }^{132}$

B. Bilki, ${ }^{132, \text { hhh }}$ W. Clarida, ${ }^{132}$ F. Duru, ${ }^{132}$ S. Griffiths, ${ }^{132}$ J.-P. Merlo, ${ }^{132}$ H. Mermerkaya, ${ }^{132, \text { iii }}$ A. Mestvirishvili, ${ }^{132}$

A. Moeller, ${ }^{132}$ J. Nachtman, ${ }^{132}$ C. R. Newsom, ${ }^{132}$ E. Norbeck ${ }^{132}$ Y. Onel, ${ }^{132}$ F. Ozok, ${ }^{132, z z}$ S. Sen, ${ }^{132}$ P. Tan, ${ }^{132}$ E. Tiras, ${ }^{132}$ J. Wetzel, ${ }^{132}$ T. Yetkin, ${ }^{132}$ K. Yi, ${ }^{132}$ B. A. Barnett, ${ }^{133}$ B. Blumenfeld, ${ }^{133}$ S. Bolognesi, ${ }^{133}$ D. Fehling, ${ }^{133}$ G. Giurgiu, ${ }^{133}$ A. V. Gritsan, ${ }^{133}$ Z. J. Guo, ${ }^{133}$ G. Hu, ${ }^{133}$ P. Maksimovic, ${ }^{133}$ M. Swartz, ${ }^{133}$ A. Whitbeck, ${ }^{133}$ P. Baringer, ${ }^{134}$ A. Bean,${ }^{134}$ G. Benelli, ${ }^{134}$ R. P. Kenny Iii, ${ }^{134}$ M. Murray, ${ }^{134}$ D. Noonan, ${ }^{134}$ S. Sanders, ${ }^{134}$ R. Stringer, ${ }^{134}$ G. Tinti, ${ }^{134}$ J. S. Wood, ${ }^{134}$ A. F. Barfuss, ${ }^{135}$ T. Bolton, ${ }^{135}$ I. Chakaberia, ${ }^{135}$ A. Ivanov, ${ }^{135}$ S. Khalil, ${ }^{135}$ M. Makouski, ${ }^{135}$ Y. Maravin, ${ }^{135}$ S. Shrestha, ${ }^{135}$ I. Svintradze, ${ }^{135}$ J. Gronberg, ${ }^{136}$ D. Lange, ${ }^{136}$ F. Rebassoo, ${ }^{136}$ D. Wright, ${ }^{136}$ A. Baden, ${ }^{137}$ B. Calvert, ${ }^{137}$ S. C. Eno, ${ }^{137}$ J. A. Gomez, ${ }^{137}$ N. J. Hadley, ${ }^{137}$ R. G. Kellogg,,${ }^{137}$ M. Kirn,,${ }^{137}$ T. Kolberg, ${ }^{137}$ Y. Lu, ${ }^{137}$ M. Marionneau ${ }^{137}$ A. C. Mignerey, ${ }^{137}$ K. Pedro, ${ }^{137}$ A. Peterman, ${ }^{137}$ A. Skuja, ${ }^{137}$

J. Temple, ${ }^{137}$ M. B. Tonjes, ${ }^{137}$ S. C. Tonwar, ${ }^{137}$ A. Apyan, ${ }^{138}$ G. Bauer,${ }^{138}$ J. Bendavid, ${ }^{138}$ W. Busza, ${ }^{138}$ E. Butz, ${ }^{138}$

I. A. Cali, ${ }^{138}$ M. Chan, ${ }^{138}$ V. Dutta, ${ }^{138}$ G. Gomez Ceballos, ${ }^{138}$ M. Goncharov, ${ }^{138}$ Y. Kim,${ }^{138}$ M. Klute,,${ }^{138}$

K. Krajczar, ${ }^{138, j j j}$ A. Levin, ${ }^{138}$ P. D. Luckey, ${ }^{138}$ T. Ma ${ }^{138}$ S. Nahn, ${ }^{138}$ C. Paus,${ }^{138}$ D. Ralph,${ }^{138}$ C. Roland, ${ }^{138}$

G. Roland, ${ }^{138}$ M. Rudolph, ${ }^{138}$ G. S. F. Stephans, ${ }^{138}$ F. Stöckli, ${ }^{138}$ K. Sumorok, ${ }^{138}$ K. Sung, ${ }^{138}$ D. Velicanu, ${ }^{138}$

E. A. Wenger, ${ }^{138}$ R. Wolf,,${ }^{138}$ B. Wyslouch, ${ }^{138}$ M. Yang, ${ }^{138}$ Y. Yilmaz, ${ }^{138}$ A. S. Yoon, ${ }^{138}$ M. Zanetti, ${ }^{138}$ V. Zhukova, ${ }^{138}$

B. Dahmes, ${ }^{139}$ A. De Benedetti, ${ }^{139}$ G. Franzoni, ${ }^{139}$ A. Gude, ${ }^{139}$ S. C. Kao, ${ }^{139}$ K. Klapoetke, ${ }^{139}$ Y. Kubota, ${ }^{139}$ J. Mans ${ }^{139}$ N. Pastika ${ }^{139}$ R. Rusack,${ }^{139}$ M. Sasseville, ${ }^{139}$ A. Singovsky,${ }^{139}$ N. Tambe, ${ }^{139}$ J. Turkewitz, ${ }^{139}$ L. M. Cremaldi, ${ }^{140}$ R. Kroeger, ${ }^{140}$ L. Perera, ${ }^{140}$ R. Rahmat, ${ }^{140}$ D. A. Sanders, ${ }^{140}$ E. Avdeeva, ${ }^{141}$ K. Bloom, ${ }^{141}$

S. Bose, ${ }^{141}$ D. R. Claes, ${ }^{141}$ A. Dominguez, ${ }^{141}$ M. Eads, ${ }^{141}$ J. Keller, ${ }^{141}$ I. Kravchenko, ${ }^{141}$ J. Lazo-Flores, ${ }^{141}$

S. Malik, ${ }^{141}$ G. R. Snow, ${ }^{141}$ A. Godshalk, ${ }^{142}$ I. Iashvili, ${ }^{142}$ S. Jain, ${ }^{142}$ A. Kharchilava, ${ }^{142}$ A. Kumar, ${ }^{142}$

S. Rappoccio ${ }^{142}$ Z. Wan, ${ }^{142}$ G. Alverson, ${ }^{143}$ E. Barberis, ${ }^{143}$ D. Baumgartel,,${ }^{143}$ M. Chasco, ${ }^{143}$ J. Haley, ${ }^{143}$ D. Nash,${ }^{143}$ T. Orimoto, ${ }^{143}$ D. Trocino, ${ }^{143}$ D. Wood, ${ }^{143}$ J. Zhang, ${ }^{143}$ A. Anastassov, ${ }^{144}$ K. A. Hahn, ${ }^{144}$ A. Kubik, ${ }^{144}$ L. Lusito, ${ }^{144}$

N. Mucia ${ }^{144}$ N. Odell, ${ }^{144}$ R. A. Ofierzynski, ${ }^{144}$ B. Pollack, ${ }^{144}$ A. Pozdnyakov,${ }^{144}$ M. Schmitt,${ }^{144}$ S. Stoynev, ${ }^{144}$

M. Velasco, ${ }^{144}$ S. Won,${ }^{144}$ D. Berry, ${ }^{145}$ A. Brinkerhoff, ${ }^{145}$ K. M. Chan, ${ }^{145}$ M. Hildreth, ${ }^{145}$ C. Jessop, ${ }^{145}$

D. J. Karmgard, ${ }^{145}$ J. Kolb, ${ }^{145}$ K. Lannon, ${ }^{145}$ W. Luo, ${ }^{145}$ S. Lynch, ${ }^{145}$ N. Marinelli, ${ }^{145}$ D. M. Morse, ${ }^{145}$ T. Pearson, ${ }^{145}$ M. Planer, ${ }^{145}$ R. Ruchti, ${ }^{145}$ J. Slaunwhite,${ }^{145}$ N. Valls, ${ }^{145}$ M. Wayne,${ }^{145}$ M. Wolf, ${ }^{145}$ L. Antonelli, ${ }^{146}$ B. Bylsma, ${ }^{146}$ L. S. Durkin, ${ }^{146}$ C. Hill, ${ }^{146}$ R. Hughes, ${ }^{146}$ K. Kotov, ${ }^{146}$ T. Y. Ling, ${ }^{146}$ D. Puigh, ${ }^{146}$ M. Rodenburg, ${ }^{146}$ C. Vuosalo, ${ }^{146}$ G. Williams, ${ }^{146}$ B. L. Winer ${ }^{146}$ E. Berry, ${ }^{147}$ P. Elmer, ${ }^{147}$ V. Halyo, ${ }^{147}$ P. Hebda,${ }^{147}$ J. Hegeman, ${ }^{147}$ A. Hunt, ${ }^{147}$ P. Jindal, ${ }^{147}$ S. A. Koay, ${ }^{147}$ D. Lopes Pegna, ${ }^{147}$ P. Lujan, ${ }^{147}$ D. Marlow, ${ }^{147}$ T. Medvedeva, ${ }^{147}$ M. Mooney, ${ }^{147}$ J. Olsen, ${ }^{147}$ P. Piroué, ${ }^{147}$ X. Quan, ${ }^{147}$ A. Raval, ${ }^{147}$ H. Saka, ${ }^{147}$ D. Stickland, ${ }^{147}$ C. Tully, ${ }^{147}$ J. S. Werner, ${ }^{147}$ S. C. Zenz ${ }^{147}$ A. Zuranski, ${ }^{147}$ E. Brownson, ${ }^{148}$ A. Lopez, ${ }^{148}$ H. Mendez, ${ }^{148}$ J. E. Ramirez Vargas, ${ }^{148}$ E. Alagoz, ${ }^{149}$ V.E. Barnes, ${ }^{149}$ D. Benedetti, ${ }^{149}$ G. Bolla,${ }^{149}$ D. Bortoletto, ${ }^{149}$ M. De Mattia, ${ }^{149}$ A. Everett, ${ }^{149}$ Z. Hu, ${ }^{149}$ M. Jones,${ }^{149}$ O. Koybasi, ${ }^{149}$ M. Kress, ${ }^{149}$ A. T. Laasanen, ${ }^{149}$ N. Leonardo, ${ }^{149}$ V. Maroussov, ${ }^{149}$ P. Merkel, ${ }^{149}$ D. H. Miller,${ }^{149}$ 
N. Neumeister, ${ }^{149}$ I. Shipsey, ${ }^{149}$ D. Silvers, ${ }^{149}$ A. Svyatkovskiy, ${ }^{149}$ M. Vidal Marono, ${ }^{149}$ H. D. Yoo, ${ }^{149}$ J. Zablocki, ${ }^{149}$ Y. Zheng, ${ }^{149}$ S. Guragain ${ }^{150}$ N. Parashar, ${ }^{150}$ A. Adair, ${ }^{151}$ B. Akgun, ${ }^{151}$ C. Boulahouache, ${ }^{151}$ K. M. Ecklund, ${ }^{151}$ F. J. M. Geurts, ${ }^{151}$ W. Li, ${ }^{151}$ B. P. Padley, ${ }^{151}$ R. Redjimi, ${ }^{151}$ J. Roberts, ${ }^{151}$ J. Zabel, ${ }^{151}$ B. Betchart, ${ }^{152}$ A. Bodek, ${ }^{152}$ Y. S. Chung, ${ }^{152}$ R. Covarelli, ${ }^{152}$ P. de Barbaro, ${ }^{152}$ R. Demina, ${ }^{152}$ Y. Eshaq, ${ }^{152}$ T. Ferbel, ${ }^{152}$ A. Garcia-Bellido, ${ }^{152}$ P. Goldenzweig, ${ }^{152}$ J. Han, ${ }^{152}$ A. Harel, ${ }^{152}$ D. C. Miner, ${ }^{152}$ D. Vishnevskiy, ${ }^{152}$ M. Zielinski, ${ }^{152}$ A. Bhatti, ${ }^{153}$ R. Ciesielski, ${ }^{153}$ L. Demortier, ${ }^{153}$ K. Goulianos, ${ }^{153}$ G. Lungu,${ }^{153}$ S. Malik, ${ }^{153}$ C. Mesropian, ${ }^{153}$ S. Arora, ${ }^{154}$ A. Barker ${ }^{154}$ J. P. Chou, ${ }^{154}$ C. Contreras-Campana, ${ }^{154}$ E. Contreras-Campana, ${ }^{154}$ D. Duggan, ${ }^{154}$ D. Ferencek,${ }^{154}$ Y. Gershtein, ${ }^{154}$ R. Gray, ${ }^{154}$ E. Halkiadakis ${ }^{154}$ D. Hidas, ${ }^{154}$ A. Lath, ${ }^{154}$ S. Panwalkar, ${ }^{154}$ M. Park,${ }^{154}$ R. Patel, ${ }^{154}$ V. Rekovic, ${ }^{154}$ J. Robles, ${ }^{154}$ K. Rose, ${ }^{154}$ S. Salur, ${ }^{154}$ S. Schnetzer,${ }^{154}$ C. Seitz,${ }^{154}$ S. Somalwar, ${ }^{154}$ R. Stone,${ }^{154}$ S. Thomas, ${ }^{154}$ M. Walker, ${ }^{154}$ G. Cerizza, ${ }^{155}$ M. Hollingsworth, ${ }^{155}$ S. Spanier, ${ }^{155}$ Z. C. Yang, ${ }^{155}$ A. York, ${ }^{155}$ R. Eusebi, ${ }^{156}$ W. Flanagan, ${ }^{156}$ J. Gilmore, ${ }^{156}$ T. Kamon, ${ }^{156, k k k}$ V. Khotilovich,${ }^{156}$ R. Montalvo, ${ }^{156}$ I. Osipenkov, ${ }^{156}$ Y. Pakhotin, ${ }^{156}$ A. Perloff, ${ }^{156}$ J. Roe, ${ }^{156}$ A. Safonov, ${ }^{156}$ T. Sakuma, ${ }^{156}$ S. Sengupta, ${ }^{156}$ I. Suarez, ${ }^{156}$ A. Tatarinov, ${ }^{156}$ D. Toback, ${ }^{156}$ N. Akchurin, ${ }^{157}$ J. Damgov, ${ }^{157}$ C. Dragoiu, ${ }^{157}$ P. R. Dudero, ${ }^{157}$ C. Jeong, ${ }^{157}$ K. Kovitanggoon, ${ }^{157}$ S. W. Lee, ${ }^{157}$ T. Libeiro, ${ }^{157}$ I. Volobouev, ${ }^{157}$ E. Appelt, ${ }^{158}$ A. G. Delannoy, ${ }^{158}$ C. Florez, ${ }^{158}$ S. Greene, ${ }^{158}$ A. Gurrola, ${ }^{158}$ W. Johns, ${ }^{158}$ P. Kurt, ${ }^{158}$ C. Maguire,,${ }^{158}$ A. Melo, ${ }^{158}$ M. Sharma, ${ }^{158}$ P. Sheldon, ${ }^{158}$ B. Snook, ${ }^{158}$ S. Tuo, ${ }^{158}$ J. Velkovska, ${ }^{158}$ M. W. Arenton, ${ }^{159}$ M. Balazs, ${ }^{159}$ S. Boutle, ${ }^{159}$ B. Cox,${ }^{159}$ B. Francis, ${ }^{159}$ J. Goodell, ${ }^{159}$ R. Hirosky, ${ }^{159}$ A. Ledovskoy, ${ }^{159}$ C. Lin,${ }^{159}$ C. Neu, ${ }^{159}$ J. Wood, ${ }^{159}$ S. Gollapinni, ${ }^{160}$ R. Harr, ${ }^{160}$ P. E. Karchin, ${ }^{160}$ C. Kottachchi Kankanamge Don, ${ }^{160}$ P. Lamichhane, ${ }^{160}$ A. Sakharov,${ }^{160}$ M. Anderson, ${ }^{161}$ Donald A. Belknap, ${ }^{161}$ L. Borrello, ${ }^{161}$ D. Carlsmith, ${ }^{161}$ M. Cepeda ${ }^{161}$ S. Dasu, ${ }^{161}$ E. Friis, ${ }^{161}$ L. Gray, ${ }^{161}$ K. S. Grogg, ${ }^{161}$ M. Grothe,${ }^{161}$ R. Hall-Wilton, ${ }^{161}$ M. Herndon, ${ }^{161}$ A. Hervé, ${ }^{161}$ P. Klabbers, ${ }^{161}$ J. Klukas, ${ }^{161}$ A. Lanaro, ${ }^{161}$ C. Lazaridis, ${ }^{161}$ R. Loveless, ${ }^{161}$ A. Mohapatra, ${ }^{161}$ M. U. Mozer, ${ }^{161}$ I. Ojalvo, ${ }^{161}$ F. Palmonari, ${ }^{161}$ G. A. Pierro, ${ }^{161}$ I. Ross, ${ }^{161}$ A. Savin, ${ }^{161}$ W. H. Smith, ${ }^{161}$ and J. Swanson ${ }^{161}$

(CMS Collaboration)

\author{
${ }^{1}$ Yerevan Physics Institute, Yerevan, Armenia \\ ${ }^{2}$ Institut für Hochenergiephysik der OeAW, Wien, Austria \\ ${ }^{3}$ National Centre for Particle and High Energy Physics, Minsk, Belarus \\ ${ }^{4}$ Universiteit Antwerpen, Antwerpen, Belgium \\ ${ }^{5}$ Vrije Universiteit Brussel, Brussel, Belgium \\ ${ }^{6}$ Université Libre de Bruxelles, Bruxelles, Belgium \\ ${ }^{7}$ Ghent University, Ghent, Belgium \\ ${ }^{8}$ Université Catholique de Louvain, Louvain-la-Neuve, Belgium \\ ${ }^{9}$ Université de Mons, Mons, Belgium \\ ${ }^{10}$ Centro Brasileiro de Pesquisas Fisicas, Rio de Janeiro, Brazil \\ ${ }^{11}$ Universidade do Estado do Rio de Janeiro, Rio de Janeiro, Brazil \\ ${ }^{12 a}$ Instituto de Fisica Teorica, Sao Paulo, Brazil \\ ${ }^{12 \mathrm{~b}}$ Universidade Estadual Paulista, Sao Paulo, Brazil \\ ${ }^{13}$ Institute for Nuclear Research and Nuclear Energy, Sofia, Bulgaria \\ ${ }^{14}$ University of Sofia, Sofia, Bulgaria \\ ${ }^{15}$ Institute of High Energy Physics, Beijing, China \\ ${ }^{16}$ State Key Lab. of Nucl. Phys. and Tech., Peking University, Beijing, China \\ ${ }^{17}$ Universidad de Los Andes, Bogota, Colombia \\ ${ }^{18}$ Technical University of Split, Split, Croatia \\ ${ }^{19}$ University of Split, Split, Croatia \\ ${ }^{20}$ Institute Rudjer Boskovic, Zagreb, Croatia \\ ${ }^{21}$ University of Cyprus, Nicosia, Cyprus \\ ${ }^{22}$ Charles University, Prague, Czech Republic \\ ${ }^{23}$ Academy of Scientific Research and Technology of the Arab Republic of Egypt, \\ Egyptian Network of High Energy Physics, Cairo, Egypt \\ ${ }^{24}$ National Institute of Chemical Physics and Biophysics, Tallinn, Estonia \\ ${ }^{25}$ Department of Physics, University of Helsinki, Helsinki, Finland \\ ${ }^{26}$ Helsinki Institute of Physics, Helsinki, Finland \\ ${ }^{27}$ Lappeenranta University of Technology, Lappeenranta, Finland \\ ${ }^{28}$ DSM/IRFU, CEA/Saclay, Gif-sur-Yvette, France \\ ${ }^{29}$ Laboratoire Leprince-Ringuet, Ecole Polytechnique, IN2P3-CNRS, Palaiseau, France
}


${ }^{30}$ Institut Pluridisciplinaire Hubert Curien, Université de Strasbourg,

Université de Haute Alsace Mulhouse, CNRS/IN2P3, Strasbourg, France

${ }^{31}$ Université de Lyon, Université Claude Bernard Lyon 1, CNRS-IN2P3, Institut de Physique Nucléaire de Lyon, Villeurbanne, France

${ }^{32}$ Institute of High Energy Physics and Informatization, Tbilisi State University, Tbilisi, Georgia

${ }^{33}$ RWTH Aachen University, I, Physikalisches Institut, Aachen, Germany

${ }^{34}$ RWTH Aachen University, III, Physikalisches Institut A, Aachen, Germany

${ }^{35}$ RWTH Aachen University, III, Physikalisches Institut B, Aachen, Germany

${ }^{36}$ Deutsches Elektronen-Synchrotron, Hamburg, Germany

${ }^{37}$ University of Hamburg, Hamburg, Germany

${ }^{38}$ Institut für Experimentelle Kernphysik, Karlsruhe, Germany

${ }^{39}$ Institute of Nuclear Physics "Demokritos," Aghia Paraskevi, Greece

${ }^{40}$ University of Athens, Athens, Greece

${ }^{41}$ University of Ioánnina, Ioánnina, Greece

${ }^{42}$ KFKI Research Institute for Particle and Nuclear Physics, Budapest, Hungary

${ }^{43}$ Institute of Nuclear Research ATOMKI, Debrecen, Hungary

${ }^{44}$ University of Debrecen, Debrecen, Hungary

${ }^{45}$ Panjab University, Chandigarh, India

${ }^{46}$ University of Delhi, Delhi, India

${ }^{47}$ Saha Institute of Nuclear Physics, Kolkata, India

${ }^{48}$ Bhabha Atomic Research Centre, Mumbai, India

${ }^{49}$ Tata Institute of Fundamental Research-EHEP, Mumbai, India

${ }^{50}$ Tata Institute of Fundamental Research-HECR, Mumbai, India

${ }^{51}$ Institute for Research in Fundamental Sciences (IPM), Tehran, Iran

${ }^{52 a}$ INFN Sezione di Bari, Bari, Italy

${ }^{52 \mathrm{~b}}$ Università di Bari, Bari, Italy

${ }^{52 \mathrm{c}}$ Politecnico di Bari, Bari, Italy

${ }^{53 a}$ INFN Sezione di Bologna, Bologna, Italy

${ }^{53 \mathrm{~b}}$ Università di Bologna, Bologna, Italy

${ }^{54 a}$ INFN Sezione di Catania, Catania, Italy

${ }^{54 \mathrm{~b}}$ Università di Catania, Catania, Italy

${ }^{55}$ a $I N F N$ Sezione di Firenze, Firenze, Italy

${ }^{55 \mathrm{~b}}$ Università di Firenze, Firenze, Italy

${ }^{56}$ INFN Laboratori Nazionali di Frascati, Frascati, Italy

${ }^{57}$ INFN Sezione di Genova, Genova, Italy

${ }^{57 b}$ Università di Genova, Genova, Italy

${ }^{58 \mathrm{a}}$ INFN Sezione di Milano-Bicocca, Milano, Italy

${ }^{58 \mathrm{~b}}$ Università di Milano-Bicocca, Milano, Italy

${ }^{59 a}$ INFN Sezione di Napoli, Napoli, Italy

${ }^{59 \mathrm{~b}}$ Università di Napoli "Federico II", Napoli, Italy

${ }^{60 \mathrm{a} I N F N}$ Sezione di Padova, Padova, Italy

${ }^{60 \mathrm{~b}}$ Università di Padova, Padova, Italy

${ }^{60 \mathrm{c}}$ Università di Trento (Trento), Padova, Italy

${ }^{61 \mathrm{a} I N F N}$ Sezione di Pavia, Pavia, Italy

${ }^{61 \mathrm{~b}}$ Università di Pavia, Pavia, Italy

${ }^{62 \mathrm{a}}$ INFN Sezione di Perugia, Perugia, Italy

${ }^{62 \mathrm{~b}}$ Università di Perugia, Perugia, Italy

${ }^{63 a}$ INFN Sezione di Pisa, Pisa, Italy

${ }^{63 \mathrm{~b}}$ Università di Pisa, Pisa, Italy

${ }^{63 \mathrm{c} S c u o l a}$ Normale Superiore di Pisa, Pisa, Italy

${ }^{64 a}$ INFN Sezione di Roma, Roma, Italy

${ }^{64 \mathrm{~b}}$ Università di Roma, Roma, Italy

${ }^{65 a}$ INFN Sezione di Torino, Torino, Italy

${ }^{65 \mathrm{~b}}$ Università di Torino, Torino, Italy

${ }^{65 \mathrm{c}}$ Università del Piemonte Orientale (Novara), Torino, Italy

${ }^{66 a}$ INFN Sezione di Trieste, Trieste, Italy

${ }^{66 \mathrm{~b}}$ Università di Trieste, Trieste, Italy

${ }^{67}$ Kangwon National University, Chunchon, Korea

${ }^{68}$ Kyungpook National University, Daegu, Korea

${ }^{69}$ Chonnam National University, Institute for Universe and Elementary Particles, Kwangju, Korea

${ }^{70}$ Korea University, Seoul, Korea

${ }^{71}$ University of Seoul, Seoul, Korea 
${ }^{72}$ Sungkyunkwan University, Suwon, Korea

${ }^{73}$ Vilnius University, Vilnius, Lithuania

${ }^{74}$ Centro de Investigacion y de Estudios Avanzados del IPN, Mexico City, Mexico

${ }^{75}$ Universidad Iberoamericana, Mexico City, Mexico

${ }^{76}$ Benemerita Universidad Autonoma de Puebla, Puebla, Mexico

${ }^{77}$ Universidad Autónoma de San Luis Potosí, San Luis Potosí, Mexico

${ }^{78}$ University of Auckland, Auckland, New Zealand

${ }^{79}$ University of Canterbury, Christchurch, New Zealand

${ }^{80}$ National Centre for Physics, Quaid-I-Azam University, Islamabad, Pakistan

${ }^{81}$ National Centre for Nuclear Research, Swierk, Poland

${ }^{82}$ Institute of Experimental Physics, Faculty of Physics, University of Warsaw, Warsaw, Poland

${ }^{83}$ Laboratório de Instrumentação e Física Experimental de Partículas, Lisboa, Portugal

${ }^{84}$ Joint Institute for Nuclear Research, Dubna, Russia

${ }^{85}$ Petersburg Nuclear Physics Institute, Gatchina (St. Petersburg), Russia

${ }^{86}$ Institute for Nuclear Research, Moscow, Russia

${ }^{87}$ Institute for Theoretical and Experimental Physics, Moscow, Russia

${ }^{88}$ Moscow State University, Moscow, Russia

${ }^{89}$ P.N. Lebedev Physical Institute, Moscow, Russia

${ }^{90}$ State Research Center of Russian Federation, Institute for High Energy Physics, Protvino, Russia

${ }^{91}$ University of Belgrade, Faculty of Physics and Vinca Institute of Nuclear Sciences, Belgrade, Serbia

${ }^{92}$ Centro de Investigaciones Energéticas Medioambientales y Tecnológicas (CIEMAT), Madrid, Spain

${ }^{93}$ Universidad Autónoma de Madrid, Madrid, Spain

${ }^{94}$ Universidad de Oviedo, Oviedo, Spain

${ }^{95}$ Instituto de Física de Cantabria (IFCA), CSIC-Universidad de Cantabria, Santander, Spain

${ }^{96}$ CERN, European Organization for Nuclear Research, Geneva, Switzerland

${ }_{97}$ Paul Scherrer Institut, Villigen, Switzerland

${ }^{98}$ Institute for Particle Physics, ETH Zurich, Zurich, Switzerland

${ }^{99}$ Universität Zürich, Zurich, Switzerland

${ }^{100}$ National Central University, Chung-Li, Taiwan

${ }^{101}$ National Taiwan University (NTU), Taipei, Taiwan

${ }^{102}$ Chulalongkorn University, Bangkok, Thailand

${ }^{103}$ Cukurova University, Adana, Turkey

${ }^{104}$ Middle East Technical University, Physics Department, Ankara, Turkey

${ }^{105}$ Bogazici University, Istanbul, Turkey

${ }^{106}$ Istanbul Technical University, Istanbul, Turkey

${ }^{107}$ National Scientific Center, Kharkov Institute of Physics and Technology, Kharkov, Ukraine

${ }^{108}$ University of Bristol, Bristol, United Kingdom

${ }^{109}$ Rutherford Appleton Laboratory, Didcot, United Kingdom

${ }^{110}$ Imperial College, London, United Kingdom

${ }^{111}$ Brunel University, Uxbridge, United Kingdom

${ }^{112}$ Baylor University, Waco, Texas, USA

${ }^{113}$ The University of Alabama, Tuscaloosa, Alabama, USA

${ }^{114}$ Boston University, Boston, Massachusetts, USA

${ }^{115}$ Brown University, Providence, Rhode Island, USA

${ }^{116}$ University of California, Davis, Davis, California, USA

${ }^{117}$ University of California, Los Angeles, Los Angeles, California, USA

${ }^{118}$ University of California, Riverside, Riverside, California, USA

${ }^{119}$ University of California, San Diego, La Jolla, California, USA

${ }^{120}$ University of California, Santa Barbara, Santa Barbara, California, USA

${ }^{121}$ California Institute of Technology, Pasadena, California, USA

${ }^{122}$ Carnegie Mellon University, Pittsburgh, Pennsylvania, USA

${ }^{123}$ University of Colorado at Boulder, Boulder, Colorado, USA

${ }^{124}$ Cornell University, Ithaca, New York, USA

${ }^{125}$ Fairfield University, Fairfield, Connecticut, USA

${ }^{126}$ Fermi National Accelerator Laboratory, Batavia, Illinois, USA

${ }^{127}$ University of Florida, Gainesville, Florida, USA

${ }^{128}$ Florida International University, Miami, Florida, USA

${ }^{129}$ Florida State University, Tallahassee, Florida, USA

${ }^{130}$ Florida Institute of Technology, Melbourne, Florida, USA

${ }^{131}$ University of Illinois at Chicago (UIC), Chicago, Illinois, USA

${ }^{132}$ The University of Iowa, Iowa City, Iowa, USA 
${ }^{133}$ Johns Hopkins University, Baltimore, Maryland, USA

${ }^{134}$ The University of Kansas, Lawrence, Kansas, USA

${ }^{135}$ Kansas State University, Manhattan, Kansas, USA

${ }^{136}$ Lawrence Livermore National Laboratory, Livermore, California, USA

${ }^{137}$ University of Maryland, College Park, Maryland, USA

${ }^{138}$ Massachusetts Institute of Technology, Cambridge, Massachusetts, USA

${ }^{139}$ University of Minnesota, Minneapolis, Minnesota, USA

${ }^{140}$ University of Mississippi, Oxford, Mississippi, USA

${ }^{141}$ University of Nebraska-Lincoln, Lincoln, Nebraska, USA

${ }^{142}$ State University of New York at Buffalo, Buffalo, New York, USA

${ }^{143}$ Northeastern University, Boston, Massachusetts, USA

${ }^{144}$ Northwestern University, Evanston, Illinois, USA

${ }^{145}$ University of Notre Dame, Notre Dame, Indiana, USA

${ }^{146}$ The Ohio State University, Columbus, Ohio, USA

${ }^{147}$ Princeton University, Princeton, New Jersey, USA

${ }^{148}$ University of Puerto Rico, Mayaguez, Puerto Rico

${ }^{149}$ Purdue University, West Lafayette, Indiana, USA

${ }^{150}$ Purdue University Calumet, Hammond, Indiana, USA

${ }^{151}$ Rice University, Houston, Texas, USA

${ }^{152}$ University of Rochester, Rochester, New York, USA

${ }^{153}$ The Rockefeller University, New York, New York, USA

${ }^{154}$ Rutgers, the State University of New Jersey, Piscataway, New Jersey, USA

${ }^{155}$ University of Tennessee, Knoxville, Tennessee, USA

${ }^{156}$ Texas A\&M University, College Station, Texas, USA

${ }^{157}$ Texas Tech University, Lubbock, Texas, USA

${ }^{158}$ Vanderbilt University, Nashville, Tennessee, USA

${ }^{159}$ University of Virginia, Charlottesville, Virginia, USA

${ }^{160}$ Wayne State University, Detroit, Michigan, USA

${ }^{161}$ University of Wisconsin, Madison, Wisconsin, USA

${ }^{\mathrm{a}}$ Deceased.

${ }^{\mathrm{b}}$ Also at Vienna University of Technology, Vienna, Austria.

${ }^{\mathrm{c}}$ Also at CERN, European Organization for Nuclear Research, Geneva, Switzerland.

${ }^{\mathrm{d}}$ Also at National Institute of Chemical Physics and Biophysics, Tallinn, Estonia.

${ }^{\mathrm{e}}$ Also at Universidade Federal do ABC, Santo Andre, Brazil.

${ }_{\mathrm{f}}^{\mathrm{f}}$ Also at California Institute of Technology, Pasadena, CA, USA.

${ }^{\mathrm{g}}$ Also at Laboratoire Leprince-Ringuet, Ecole Polytechnique, IN2P3-CNRS, Palaiseau, France.

${ }^{\mathrm{h}}$ Also at Suez Canal University, Suez, Egypt.

${ }^{\mathrm{i}}$ Also at Zewail City of Science and Technology, Zewail, Egypt.

${ }^{\mathrm{j}}$ Also at Cairo University, Cairo, Egypt.

${ }^{\mathrm{k}}$ Also at Fayoum University, El-Fayoum, Egypt.

${ }^{1}$ Also at British University in Egypt, Cairo, Egypt.

${ }^{\mathrm{m}}$ Now at Ain Shams University, Cairo, Egypt.

${ }^{\mathrm{n}}$ Also at National Centre for Nuclear Research, Swierk, Poland.

${ }^{\circ}$ Also at Université de Haute Alsace, Mulhouse, France.

${ }^{\mathrm{P}}$ Also at Joint Institute for Nuclear Research, Dubna, Russia.

${ }^{\mathrm{q}}$ Also at Moscow State University, Moscow, Russia.

${ }^{\mathrm{r}}$ Also at Brandenburg University of Technology, Cottbus, Germany.

${ }^{\mathrm{s}}$ Also at The University of Kansas, Lawrence, KS, USA.

${ }^{\mathrm{t}}$ Also at Institute of Nuclear Research ATOMKI, Debrecen, Hungary.

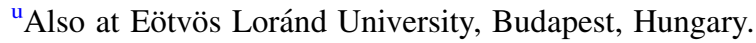

${ }^{v}$ Also at Tata Institute of Fundamental Research - HECR, Mumbai, India.

${ }^{w}$ Now at King Abdulaziz University, Jeddah, Saudi Arabia.

${ }^{\mathrm{x}}$ Also at University of Visva-Bharati, Santiniketan, India.

${ }^{\mathrm{y}}$ Also at Sharif University of Technology, Tehran, Iran.

${ }^{\mathrm{z}}$ Also at Isfahan University of Technology, Isfahan, Iran.

${ }^{\text {aa } A l s o ~ a t ~ S h i r a z ~ U n i v e r s i t y, ~ S h i r a z, ~ I r a n . ~}$

${ }^{\mathrm{bb}}$ Also at Plasma Physics Research Center, Science and Research Branch, Islamic Azad University, Tehran, Iran. 
${ }^{\mathrm{cc}}$ Also at Facoltà Ingegneria, Università di Roma, Roma, Italy.

${ }^{\mathrm{dd}}$ Also at Università della Basilicata, Potenza, Italy.

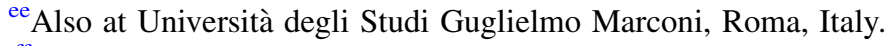

${ }^{\mathrm{ff}}$ Also at Università degli Studi di Siena, Siena, Italy.

${ }^{g g}$ Also at University of Bucharest, Faculty of Physics, Bucuresti-Magurele, Romania.

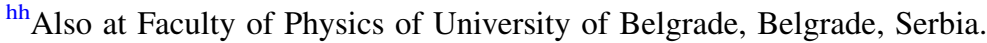

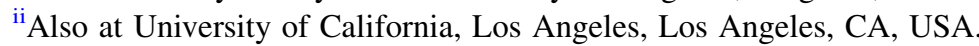

${ }^{\mathrm{jj}}$ Also at Scuola Normale e Sezione dell'INFN, Pisa, Italy.

${ }^{\mathrm{kk}}$ Also at INFN Sezione di Roma, Roma, Italy.

${ }^{11}$ Also at University of Athens, Athens, Greece.

${ }^{\mathrm{mm}}$ Also at Rutherford Appleton Laboratory, Didcot, United Kingdom.

${ }^{\mathrm{nn}}$ Also at Paul Scherrer Institut, Villigen, Switzerland.

${ }^{\circ o}$ Also at Institute for Theoretical and Experimental Physics, Moscow, Russia.

${ }^{p p}$ Also at Albert Einstein Center for Fundamental Physics, Bern, Switzerland.

${ }^{\mathrm{qq}}$ Also at Gaziosmanpasa University, Tokat, Turkey.

${ }^{\mathrm{rr}}$ Also at Adiyaman University, Adiyaman, Turkey.

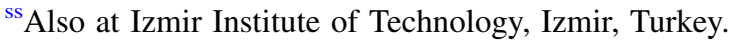

${ }^{t t}$ Also at The University of Iowa, Iowa City, IA, USA.

${ }^{\mathrm{uu}}$ Also at Mersin University, Mersin, Turkey.

${ }^{\mathrm{vv}}$ Also at Ozyegin University, Istanbul, Turkey.

${ }^{w w}$ Also at Kafkas University, Kars, Turkey.

${ }^{\mathrm{xx}}$ Also at Suleyman Demirel University, Isparta, Turkey.

${ }^{\mathrm{yy}}$ Also at Ege University, Izmir, Turkey.

${ }^{\mathrm{zz}}$ Also at Mimar Sinan University, Istanbul, Istanbul, Turkey.

${ }^{\text {aaa }}$ Also at Kahramanmaras Sütcü Imam University, Kahramanmaras, Turkey.

${ }^{\mathrm{bbb}}$ Also at School of Physics and Astronomy, University of Southampton, Southampton, United Kingdom.

${ }^{c c c}$ Also at INFN Sezione di Perugia, Università di Perugia, Perugia, Italy.

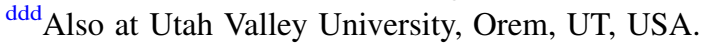

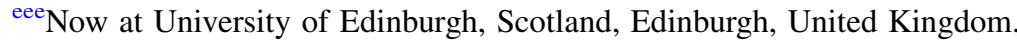

${ }^{\mathrm{fff}} \mathrm{Also}$ at Institute for Nuclear Research, Moscow, Russia.

${ }^{\text {ggg }}$ Also at University of Belgrade, Faculty of Physics and Vinca Institute of Nuclear Sciences, Belgrade, Serbia.

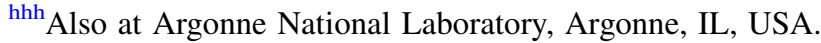

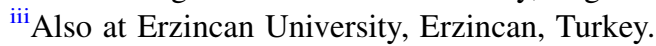

${ }^{\mathrm{jjj}} \mathrm{Also}$ at KFKI Research Institute for Particle and Nuclear Physics, Budapest, Hungary.

${ }^{k k k}$ Also at Kyungpook National University, Daegu, Korea. 